\title{
Sustancia e inherencia en las Categorías de Aristóteles*
}

\author{
FABIÁN Mí́ \\ Escuela de Filosofía \\ Universidad Nacional de Córdoba \\ Consejo Nacional de Investigaciones Científicas y Técnicas (CONICET) \\ FABIANGUSTAVOMIE@fullzero.com.ar
}

\begin{abstract}
Resumen: Las combinaciones y los tipos de relaciones posibles entre las distintas entidades que acepta la ontología de las Categorías regulan la economía ontológica de la primera metafísica aristotélica, estableciendo vínculos de prioridad y posterioridad entre las sustancias sobre la base de ciertos criterios que Aristóteles se esfuerza por clarificar. Pero el primer esencialismo aristotélico se elabora a medida que el autor de las Categorías fija una distinción entre propiedades esenciales y accidentales a fin de explicar qué es necesariamente una cosa y qué puede o no tener sin que por ello se vea afectada su propia identidad. Este texto pretende reconstruir la relación transversal entre sustancias y accidentes, perfilando la noción de accidente junto a otras de orden semántico. Esta relación, R2, se distingue de la predicación esencial, R1, que tiene lugar verticalmente entre sustancias segundas y primeras.
\end{abstract}

Palabras clave: metafísica, primer esencialismo aristotélico, reconstrucción conceptual

\begin{abstract}
The combination and possible relations between the different types of entities present in the first Aristotelian essentialism govern the ontological economy in Categories by establishing priority and posteriority links among substances (I will refer to such vertical links as R1) on the basis of some criteria that Aristotle tries to clarify. However, the first Aristotelian essentialism is grounded on a distinction between essential and accidental properties, which might contribute to explain what an object is necessarily, and what features might it have or lack without compromising its identity. In this paper I offer a reconstruction of the transversal relation (R2) among substance and accidents as well as of the Aristotelian concept of accident and of some associated semantic features.
\end{abstract}

Key words: metaphysics, first Aristotelian essentialism, conceptual reconstruction

El siguiente trabajo aborda la noción de accidente en la primera metafísica de Aristóteles; para ello se centra en una reconstrucción de las Categorías y toma como horizonte una reconstrucción y una discusión del esencialismo aristotélico. En la primera sección introduzco la noción de accidente sirviéndome de la relación que guardan los accidentes con los sujetos. Allí mismo comienzo a perfilar las principales tesis sobre dependencia ontológica que sostiene Aristóteles en las Categorías, donde se destaca el papel que asumen los sujetos genuinos y últimos en la infraestructura de la primera metafísica aristotélica. En la segunda sección distingo funciones del sujeto en las principales relaciones ontológicas admitidas en las Categorías, a fin de despejar la relación entre el accidente y el sujeto, relación que, tal

* Deseo reconocer aquí las observaciones y sugerencias que el dictaminador de Diánoia hizo a una versión anterior de este trabajo. 
como intentaré definirlo, debemos distinguir en especial de otras dos en las que entran sendas clases de partes (las partes constitutivas y las partes físicas). Además, formulo allí algunas ideas acerca de dos distintas clases de individuos que Aristóteles debería admitir en este marco, procurando destacar la incidencia que tiene tal distinción en una demarcación de lo que se admite en las Categorías como entidades básicas o sustancias primeras. En la tercera sección trato de explicar la predicación accidental en términos de "predicación nominal", e intento esclarecer la noción de accidente por apelación a los parónimos; así reviso una difundida opinión que priva a la paronimia de una contribución importante en la fundamentación de la primera metafísica de Aristóteles, sin comprometerme con una identificación errónea de los parónimos con lo que la metafísica madura del estagirita elaborará como una tesis acerca de la unidad focal del ser. Por último, en la cuarta sección me sirvo de la clarificación anterior acerca de los parónimos para sostener que la única tesis consistente con la semántica y la metafísica de las Categorías es la que admite accidentes particulares. Allí mismo trato de perfilar cómo hay que entender la individualidad de las sustancias primeras y la de los particulares no sustanciales.

\section{La relación tiene}

En Categorías (Cat.) 5, 3a7-8, Aristóteles destaca como rasgo común a toda sustancia individual y primera que esta clase de entidad no es-en un sujeto. Además, Aristóteles demarca los accidentes como entidades que inhieren en tales sustancias, de lo cual se sigue que si un accidente particular $a$ es-en $x$ como en un sujeto individual, entonces $a$ no es una propiedad dicha en común o predicada universalmente de $x$, ya que lo predicado universalmente de una sustancia no puede ser-en un sujeto. Por otro lado, en virtud del hecho de depender de un sujeto, $a$ no es una entidad primaria. Que $a$ no es una sustancia segunda está excluido por el hecho, ya mencionado, de que éstas se dicen-de un sujeto (cfr. 2, 1a20-22; 5, 3a9-15), pero no son-en ningún sujeto. Una sustancia segunda $(F)$ determina qué es un objeto $x$, constituyendo la definición del mismo o formando parte de la definición; esto confiere a las sustancias segundas una prioridad explicativa ante las sustancias primeras, aunque $F$ no puede existir independientemente de aquel $x$ que define sólo en tanto y en cuanto $x$ es $F .^{1}$

Las Categorías operan con tres tipos diferentes de partes que se incluyen en las relaciones ontológicas allí establecidas. Aclarar esas distintas nociones de parte resulta de importancia central, ya que Aristóteles fundamenta su concepto de accidente explicando esta clase de entidad como un tipo de

\footnotetext{
${ }^{1}$ Usaré aquí las notaciones es (ser) y tiene (tener) para distinguir la predicación esencial de la accidental, respectivamente, siguiendo con ello la jerga (y en buena medida también algunas sugerencias interpretativas) de Code (1986).
} 
parte diferente de los otros dos principales. La primera clase de parte se halla en R1, donde $x$ es parte constitutiva $(P c)$ de $F$, por cuanto $x$ es parte de la extensión del concepto definido por $F$. Además, $x$ es estructuralmente idéntico a $F$ - Sócrates y hombre admiten la misma definición o son esencialmente indistintos- - Una segunda noción de parte es la de parte física $(P f)$ de una sustancia individual. Veremos que en 2, 1a24, Aristóteles delimita una noción de parte atributiva $(\mathrm{Pa})$ frente a la última clase de parte mencionada. Dicho brevemente: una $P f$ es un componente material de un individuo sustancial que, como parte física del mismo, se caracteriza por aportar una condición material funcionalmente indispensable para la realización de la estructura $F$ en $x$.

El primer modelo ontológico del estagirita se caracteriza por no efectuar una fundamentación de la realización de $F$ en un $x_{i}$ determinado; sin embargo, la lógica de esta relación permite extraer algunas consecuencias para las $P f$ que tal vez sea útil tener en cuenta en una interpretación cuyo fin es reconstruir la metafísica de las Categorías en términos de una fundamentación de su esencialismo, que pronto se revelará insuficiente. Mi propuesta al respecto - aunque no me dedico a desarrollarla aquí- es que el segundo modelo ontológico de Aristóteles —desplegado en Metafísica (Metaph.) VII-IX - plantea tesis que mantienen una continuidad importante con la ontología de las Categorías, sin que ello implique opacar las innovaciones centrales que formula y sustenta Metaph. VII-IX. Mi idea es que algunas de esas innovaciones tienen como punto de partida la resolución de ciertos problemas que suscita la misma consideración del papel que desempeñan las partes físicas de una sustancia en la constitución de la identidad mediante la determinación esencial de una sustancia a través de una propiedad cuyo antecedente en el modelo de las Categorías está dado por las sustancias segundas. Sin embargo, así como en las Categorías no se analiza la composición de una sustancia primera en términos de conceptos que permitan correlacionar la estructura esencial y sus condiciones de realización material - lo que es aportado por el par materia-forma en la metafísica madura-, la primera metafísica de Aristóteles tampoco esclarece el carácter relacional que de cierta manera está contenido en su propia noción de sustancias segundas. La consecuencia de esto es que en Categorías se registra una doble y correlativa presuposición infundada: por un lado, el sujeto de la predicación esencial se toma como una "unidad sellada", provista de una estructura interna a la que no se accede analíticamente; por otro lado, la estructura en sí se explica como una clase de entidad cuya independencia garantiza su sustancialidad. Pero esto último tiene como contrapartida que las sustancias segundas se sustraen a cualquier explicación de su aporte a la determinación de la estructura que exhibe una sustancia individual. En resumen, la primera metafísica de Aristóteles no fundamenta un supuesto central del esencialismo: la atribución a los objetos de una es- 
tructura esencial; y, así, postula una duplicación de objetos o dos clases de sustancias, que reproducen, a su manera, el problema platónico de los dos mundos, el cual consiste en la falta de explicación del vínculo que guardan entre sí las formas y los particulares sensibles. La primera metafísica de Aristóteles se propone solucionar esta dificultad en una de sus aristas invirtiendo el orden de las prioridades ontológicas a través de la tesis del carácter básico de los individuos sustanciales y por contraposición a la anterioridad de que las formas platónicas estaban dotadas en la metafísica de las ideas. En efecto, la única herramienta teórica de que Aristóteles dispone en su primer modelo ontológico para dar cuenta de la relación entre sustancias primeras y segundas está estrechamente emparentada con aquella que, según el mismo estagirita, llevó a Platón a fracasar con la noción de "participación". Tal herramienta es la predicación, y, por cierto, una noción de predicación que relaciona semánticamente sujeto y predicado, ${ }^{2}$ es decir, sin introducir el problema de la relación entre una estructura y un objeto real estructurado. Si esto es así, la noción de $P f$ en las Categorías demarca un punto límite, un problema en la fundamentación del primer modelo ontológico aristotélico, modelo que aquí me propongo considerar con vistas a individualizar tópicos que debería incluir una metafísica esencialista adecuadamente fundamentada, tal como la que Aristóteles despliega en su madurez.

Si bien, como advertí, no abordaré ahora el traspaso y la conexión entre el primero y el segundo modelo ontológico del estagirita, la consideración de ciertas implicaciones contenidas en la noción de $P f$, que Aristóteles debería aceptar, entraña un aporte para mi propósito reconstructivo del primero de esos modelos. Por ejemplo, por el mismo motivo de que una $P f$ constituye una condición para la realización de $F$ en $x_{i}$, una $P f$ determinada $\left(P f_{i}\right)$ debe ser funcionalmente reemplazable. Además, en cuanto una $P f$ compone materialmente al sujeto sustancial, ella parece poder separarse de él sin perder su condición de componente material de $x$. Una mano $(m)$ de Sócrates puede separarse del cuerpo total de ese individuo que la mano compone materialmente; sin embargo, esa separación física no le impediría a la $P f$ mantener sólo hasta cierto punto la determinación por la cual ese pedazo de distintos tejidos es una mano,

\footnotetext{
${ }^{2}$ Me refiero con esto a que los universales sustanciales merecen esa calificación por el hecho de que dan el qué es (tí estin) de un individuo sustancial (cfr., e.g., Cat. 5, 2b32), con lo cual surge la necesidad de distinguir entre una determinación más lejana o amplia y común a muchos individuos de un mismo género, y otra más cercana y definida con respecto a un individuo. Por medio de esta última determinación se da una precisión mayor acerca de la estructura propia de un individuo, razón por la cual se diferencian específicamente dos individuos que comparten un mismo género (2b7-17). Pero el vínculo entre género-especie y la sustancia que concibe este primer modelo ontológico reside y se acaba en esa determinación de la estructura de la segunda por medio de una forma que no es ni más ni menos que la propiedad nombrada en un predicado esencial de un individuo.
} 
pues un trozo de cuerpo amputado está sometido a una corrupción rápida derivada de que ya no es parte del sustrato que realiza la forma del caso; por ejemplo, la forma de hombre. De esa pérdida de la forma se sigue que cierto trozo de carne amputado no puede desempeñar ya las funciones orgánicas para las cuales su constitución fisiológica y disposición anatómica interna estaban previstas. ${ }^{3}$ Dado, entonces, que un trozo de materia cualquiera adquiere su determinación como $P f$ de una sustancia a partir de su vinculación funcional con cierto sujeto específicamente determinado, esa mano que ahora suponemos separada no puede considerarse estrictamente como la mano de $x .{ }^{4}$ Ligado a esta restricción se halla el hecho de que, como parte, $m$ no representa un individuo más dentro de $x$, tomado como sujeto de $F$. Una $P f$ no es un genuino sujeto sustancial $(S)$; prueba de ello es que el nombre de una $P f$ no puede sustituir al nombre de la sustancia primera — de la cual esa $P f$ es parteen un enunciado donde dicho nombre de la sustancia primera se desempeña como sujeto y el lugar del predicado está ocupado por el nombre de una sustancia segunda. De esta manera, no puede decirse, por ejemplo, "esta mano (de Sócrates) es un hombre". Curiosamente, tal como veremos, las $P f$ son una especie de sustancia en el primer modelo ontológico, pero eso no conlleva una multiplicación de sujetos primarios en

${ }^{3}$ La fundamentación de la incidencia que tienen las $P f$ en la constitución de una sustancia, así como la de la relación que guardan las $P f$ con un cuerpo sustancial considerado como un todo persistente, son cosas que requieren distinguir entre el cambio de propiedades accidentales peculiares, en el cual persiste un sustrato perceptible (alteración), por un lado, y el cambio que atañe al sustrato sensible en lo que hace a la continuidad de éste como sujeto idéntico, del cual forman parte las $P f$ (generación-corrupción). Aristóteles fundamenta esta distinción recién en De la generación y la corrupción (GC) I 4, la cual hace una contribución importante a la discusión metafísica acerca de la identidad y la persistencia sustancial. De manera interesante, el primer modelo ontológico clasifica como el rasgo más distintivo de las sustancias primeras su capacidad de admitir contrarios - lo que implica cierto cambio (cfr. Cat. 5, 4b2-4) - pero persistiendo en su unidad e identidad (4a10-11), con lo cual en dicho modelo se intuye algo acerca de las diferentes condiciones de cambio que atañen a entidades sustanciales y accidentales. Sin embargo, esta posición sólo puede avanzar hacia una clarificación de esa intuición en la medida en que efectúa un análisis hilemórfico de la sustancia primera, lo cual supone la introducción de nuevos y diferentes criterios para hablar de sustancias con relación a los vigentes en la primera metafísica de Aristóteles. Gill 1991, especialmente en los capítulos 2 y 3 , destacó con precisión la incidencia que tiene la teoría del cambio sustancial desarrollada en $G C$ con respecto a la nueva teoría de la sustancia que formula la metafísica madura del estagirita.

${ }^{4}$ Aquí no entro a discutir el análisis que hace Aristóteles de la relación entre un cuerpo vivo y un cadáver con las herramientas conceptuales del análisis hilemórfico de las entidades. $\mathrm{Al}$ respecto, uno de los resultados de tal análisis, característico de la metafísica madura del estagirita, es que el cadáver de un ser humano no es más que homónimamente un hombre, ya que no está organizado por la estructura que realiza un hombre vivo. El evaluador de este artículo llamó la atención sobre este problema y cierta ambigüedad en la formulación de la versión anterior de este trabajo. Sobre el tema cfr. Ackrill 1972-1973; para una completa discusión de las relaciones entre alma y cuerpo en Aristóteles, cfr. Hartmann 1977, capítulo cuarto; y sobre la relación materia-forma en general, cfr. Lewis 1991, pp. 143-261. 
la metafísica de las Categorías. Y esto es así porque las nociones de $P f$ y $P c$ se hallan perfectamente distinguidas en el primer esencialismo aristotélico. $^{5}$

Para comenzar a delimitar R2 podemos establecer la siguiente fórmula:

(1) si $a$ es-en $x$ como en un $S^{*},{ }^{6}$ entonces $a$ no es ni $P c$ ni $P f$ de $x$.

Cat. 1 establece distintas vinculaciones semánticas entre los ítems ligados mediante las dos relaciones principales R1 y R2. Dos cosas que se hallan en una relación donde el concepto de una da la esencia de la otra, como en la expresión " $x$ es $F$ ", se relacionan cada una de la misma manera con la determinación esencial o estructura denominada ' $F$ '. Así, Sócrates y hombre admiten la misma definición por cuanto están constituidos por la misma estructura (supongamos: animal racional). Aristóteles sostiene que un ejemplar de cierta especie y esta misma especie comparten no sólo el nombre, sino también lo que se define como estructura (lógos tês ousías) y se denomina con aquel nombre (cfr. 1a6-7). La noción de la sinonimia permite, entonces, seleccionar, recurriendo al criterio de la definición común, la clase de ítems que se hallan relacionados en la predicación esencial, y nos autoriza a caracterizarlos semánticamente como sinónimos. ${ }^{7}$

\footnotetext{
${ }^{5}$ Para una mejor explicación de esto último me veo obligado aquí a remitir a un artículo donde abordo particularmente este tema; $c f r$. Mié en prensa.

${ }^{6}$ Mediante la notación $S^{*}$ distinguiré la función de $x$ como sujeto individual sustancial de un accidente, relación donde se asume la plena determinación esencial de $x$ (como $S$ de $F$ ). Posteriormente demarcaré otras funciones de sujeto que admite Cat.; en lo inmediato, $S^{*}$ sirve para contraponer la función del sujeto de un accidente respecto de la función primaria del sujeto en la predicación esencial (R1), que es lo que hace de $x$ una sustancia primera y lo que delimita la clase de sujetos básicos en esta metafísica.

${ }^{7}$ En esta caracterización de la sinonimia, como también en las siguientes de las otras dos nociones semánticas introducidas por Cat. 1, acepto que Aristóteles clasifica primeramente cosas o ítems extralingüísticos, y que incluye expresiones del lenguaje sólo en la medida en que contribuyen a abordar su objetivo primero, que es clasificar distintos tipos de significados de los términos con que operamos ordinariamente al usar el lenguaje para hablar de cosas. Véase una defensa de esta lectura en Oehler 1997, pp. 205 s. Esta lectura evita algunos problemas en los que incurriría la sinonimia aristotélica. Es evidente que Aristóteles no pudo haber concebido nominalmente la sinonimia $(R s)$, es decir, a base del nombre $(N)$, de tal manera que $x$ sería sinónimo de $y(x R s y)$ si $(x),(y), N, N(x) \supset N(y)$. Si así hubiera sido, Aristóteles debería haber excluido que un particular sustancial (llamado, por ejemplo, Calias) y la clase a la que él mismo pertenece (hombre) pudieran ser sinónimos. Pero aunque Aristóteles no introduzca esta distinción entre miembros particulares y clase en su exposición sobre semántica en Cat. 1, él toma posteriormente a un hombre particular (ho tis ánthropos, átomon kaì hèn arithmôi, cfr. e.g. 2, 1b4, 1b6-7; 5, 2b26-28, 3b12) y al universal sustancial respectivo como sinónimos. Esto indica que Calias o Sócrates son sinónimos entre sí con respecto a hombre, no en cuanto a sus nombres, sino en cuanto a la definición de la estructura (lógos tês ousías, 1, 1a9-10) que ambos comparten con ese universal. Ésta es una tesis aristotélica tan fundamental como que sólo a base de ella se puede considerar a cierta clase de individuos como sustanciales, pues un hombre particular es ousía (cfr. e.g. 5, 2b27) sólo en virtud de que posee una y la misma estructura que define la especie correspondiente (ho ánthropos, 2b25), de tal manera que a ambos —individuo sustancial y especie- se aplica la misma definición.
} 
Frente a la sinonimia, la homonimia representa una relación no esencial entre dos cosas que sólo comparten convencionalmente el nombre, es decir, que tienen meramente el nombre en común; pero lo que significa para $x$ llamarse $H$ no es lo mismo que lo que para y significa llamarse $H$ ( $c f r$. 1a1-2).

La tercera clasificación semántica incluida en las Categorías corresponde a los ítems parónimos. En este ensayo me propongo mostrar especialmente de qué manera la paronimia le permite a Aristóteles explicar los accidentes. Entidades parónimas son las sustancias en cuanto que se consideran caracterizadas como portadoras de un accidente. 'Sócrates-justo' designa un sujeto caracterizado mediante un modo contingente de su presentación, que, por su parte, instancia una propiedad no esencial, realizándola a través de una modificación de que se da cuenta lingüísticamente a través del cambio en la terminación del nombre de aquella propiedad, en este caso, 'justicia'. ${ }^{8}$

Entonces, inicialmente podemos distinguir R1 y R2 en los siguientes términos:

(2) R1 se funda en la sinonimia entre $x / F ;{ }^{9}$ en cambio, el par $x / a$ en R2

En la predicación esencial de forma gramatical normal, del sujeto se dice el lógos y el ónoma de las sustancias segundas, cfr. e.g. 3a17-20; "Pero de las sustancias segundas se predicará del sujeto tanto la definición como el nombre" (3a17). Cosas que tienen no sólo el nombre, sino también y particularmente la definición en común son sinónimas (1, 1a6-7). Esto anticipa la vinculación entre R1 y sinonimia, que intentaré explicar a continuación. Sobre la fórmula ho katà toúnoma lógos tês ousías (algo así como "el enunciado de la esencia correspondiente al nombre (de la cosa ") cfr. Oehler 1997, pp. 201 ss.

${ }^{8}$ Más adelante, en (3), me ocupo en particular de los parónimos. Sobre las distinciones semánticas de Cat. 1, cfr. Oehler 1997, pp. 189-212.

${ }^{9}$ Para aclarar un poco más el caso de la sinonimia, cabe señalar que dicha relación está vigente entre (i) individuos (Sócrates y Calias) con respecto a la estructura común a ambos (hombre), entre (ii) especies (hombre y caballo) con respecto al género (animal), pero también entre (iii) un individuo y su especie, en cuanto que (a) el nombre específico que se aplica a Sócrates es el nombre de la clase o especie de la cual ese individuo es miembro, y en cuanto que (b) la determinación de lo que es para Sócrates ser hombre es lo que define la especie. Así, x qua $F$ es sinónimo de $F$ con relación al $N$ de ambos. Podemos entender mejor la relación de la sinonimia $(R s)$ y aplicarla también a la relación entre un individuo específicamente considerado y su especie si visualizamos esa relación semántica a través de la siguiente formalización:

$$
\begin{array}{ll}
(x),(y), x \neq y, x \text { Rs } y \text { si } & \text { (i) } x \text { y } y \text { tienen el mismo } N \\
& \text { (ii) la determinación de lo que es para } x \text { llamarse } N \text { es la misma } \\
& \text { de lo que es para } y \text { llamarse } N \text {, de tal manera que } \\
& \text { (iii) } x==_{D} y .
\end{array}
$$

En (ii) se estipula el significado constante del nombre en las dos aplicaciones distintas que se hacen de él en $N(x)$ y $N(y)$. Para una discusión relativa a algunos problemas que podrían derivarse de predicar de la especie la propiedad que ella misma define y que se atribuye a sus miembros - una cuestión conocida en la literatura como el "argumento del tercer hombre"-, cfr. Lewis 1991, pp. 51 ss. Lewis propone que la salida aristotélica al tercer hombre reside en 
no articula miembros sinónimos; por tanto, la definición de $a$ no se predica de $x$ ni $x$ es $P c$ de $a$ (cfr. 5, 2a19-34, 3a15-21).

(2) intenta comenzar a establecer las condiciones para que se admitan relaciones no esenciales entre dos ítems. Puede resultar útil pasar revista ahora a los ítems relacionados en las distintas aplicaciones de R2. La distinción radica en la cuantificación de tales ítems, como se presenta a continuación. R2 es satisfecha por los siguientes pares (cfr. Cat. 2, 1a26-28, 1b8-9, 1a2930, 1b1-2, 2a30-31, 2b1-3):

$\mathrm{R} 21$ conocimiento gramatical (particular), alma/hombre (particular)\};

$\mathrm{R} 2_{2}$ \{blanco determinado (particular), cuerpo/hombre (general)\};

$\mathrm{R} 2{ }_{3}$ \{color (general), cuerpo/hombre (general)\};

R2 4 \{conocimiento (general), alma/hombre (particular)\}.

Todas estas relaciones establecen lo que se denomina inherencia de una propiedad en un sujeto particular o general. Aristóteles no acepta que unos y otros sujetos ostenten el mismo rango; sabemos que la ontología de las Categorías ubica a los individuos sustanciales como sujetos genuinos y, en tal sentido, como las únicas entidades básicas. Antes de abordar esta cuestión es oportuno aclarar la noción de inherencia distinguiendo cuáles son las condiciones generales para que una entidad inhiera en otra.

$$
a \text { es-en } x \text { como en un } S^{*} \text { si }
$$

(i) $a$ es-en $x$ no como una $P f$

de $x$;

(ii) $a$ no puede existir separado

de $x$ qua $S .{ }^{10}$

la distinción binaria entre esto (tóde, una sustancia) y tal (poión, una propiedad), distinción según la cual todo lo que es un esto no puede ser también un tal. De esa manera, no puede generarse una serie infinita y creciente de formas por el solo hecho de que la forma o especie de hombre defina la determinación que se dice de múltiples hombres particulares, pues esa forma no es un esto del cual se predique una especie. Para la distinción esto/tal, cfr. Cat. 5, 3b10-23.

${ }^{10}$ Cat. 2, 1a24-25: "en un sujeto digo que es (i) lo que cabe en algo no como una parte y (ii) es imposible que sea separado de aquello en lo que es" (en hypokeiménoi dè légo (i) hò én tini mè hos méros hypárchon (ii) adýnaton chorì eînai toû en hôi estín). Para una discusión de este texto, cfr. Oehler 1997, pp. 216 ss.; Devereux 1992, p. 122. En contra está Frede 1987a. Para algunas pertinentes objeciones a Frede, cfr. Oehler 1997, pp. 221-225; Devereux 1992, p. 121, n. 11. Aquí defenderé que la llamada "condición de inseparabilidad", como se lee en (3ii), se interpreta de manera adecuada mediante una cláusula de inseparabilidad fuerte, según la cual un accidente no depende en su existencia y determinación meramente de algún sujeto, sino que no puede existir independientemente de aquel preciso sujeto en el cual (en hôi) contingentemente existe. Esta lectura tiene una de sus principales consecuencias en una noción de individualidad numérica de los accidentes particulares, un tema muy controvertido que aquí discutiré sólo colateralmente, ya que mi objetivo en el presente trabajo es perfilar el concepto aristotélico de inherencia como fundamento de la noción general de accidente en Aristóteles. 
(3) permite establecer dos razones para sostener que $a$ no es una sustancia. Según (3i), $a$ no es una $P f-y$ Aristóteles toma las $P f$ como partes materiales sustanciales de un sujeto que funciona como un todo en relación con sus componentes-; además, de acuerdo con (3ii), a no puede existir sin el sujeto determinado - según mi lectura - en el cual cabe. Es decir, a diferencia de los universales sustanciales $(F)$ - por ejemplo, hombre-, que existen mientras existe algún sujeto individual $(x)$ del cual es verdadero " $x$ es $F$ ", (3ii) indica que los accidentes mantienen lo que se podría llamar una dependencia particular con el sujeto individual en el cual inhieren. Si mi lectura es correcta, (3i) delimita los accidentes frente a las $P f$ y (3ii) hace lo propio pero con respecto a las $P c .{ }^{11}$

En 1a24-25, Aristóteles no formula, entonces, una definición circular de la inherencia, como algunos comentadores lo han denunciado. La aparición de la preposición 'en' dentro del definiens y del definiendum no puede tener el mismo sentido técnico en ambos casos, como lo explicó Ackrill. En el definiendum, el 'en' debe ser comprensible sin presuponer una noción terminológica; tiene que referirse, entonces, generalmente a un uso que el definiens se propone delimitar y restringir. $\mathrm{O}$ sea, Aristóteles está diciendo allí algo así como: ahora bien, hay distintas cosas que son en otras, pero cuando uso 'en' para referirme a la manera en que un accidente es-en un sujeto, debe entenderse 'en' bajo un régimen que lo delimita frente a dos casos especialmente relevantes en los cuales la misma preposición tiene un significado diverso. Y a continuación menciona esos dos casos. Para ello, Aristóteles cree necesario restringir el modo peculiar según el cual los accidentes son-en, especificando la inseparabilidad fuerte de los mismos, ya que en la inseparabilidad pueden sintetizarse las dos condiciones que incluye (3), en la medida en que ni para las $P f$ ni para las $P c$ rige la inseparabilidad que domina a los accidentes. Por cierto, con la inseparabilidad se trata de una delimitación diferente que corresponde a las dos distintas clases de entidades incluidas en 1a24-25.

En el caso de la dependencia universal, frente a la cual se demarca la clase de dependencia particular propia de los accidentes, es decir, lo que establece (3ii), se trata de delimitar las $P a$ frente a las $P c$ atendiendo al hecho de que basta con que exista un individuo sustancial, del cual vale " $x$ es $F$ ", para que $F$ pueda existir. Una consecuencia de esta tesis es que $F$ puede existir en tanto y en cuanto exista al menos un $x$ del cual vale " $F$ se dice-de $x$ como de un $S$ ". Precisamente esta condición de dependencia no particular $-F$ existe en tanto y en cuanto existe al menos uno cualquiera de los individuos de los cuales $F$ se predica - no se aplica a la relación $a R x$, ya que el contenido conceptual de a supone una relación determinada y particular (aunque contingente) con un sujeto - cuya función debe explicarse

${ }^{11}$ Devereux 1992, pp. 124 s., en cambio, toma Cat. 2, 1a25, como una glosa de 1a24. 
forzosamente como diferente de la que $x$ desempeña en FRx- La dependencia particular y determinada que articula R2 proviene necesariamente no de la esencia universal de $x$, pues $x$ no es específicamente diferente de otro sujeto $y$-suponiendo que $y$ es $F$ y y no tiene $a$-, sino que la relación de dependencia particular, característica de $a$ en $a R x$, así como la inseparabilidad fuerte que expresa (3ii) se basan en la individualidad del sujeto de las propiedades inherentes. ${ }^{12}$

La individualidad característica de los sujetos genuinos (Sócrates) de los accidentes implica, entonces, la particularidad de estos últimos. Pero esta condición de individualidad del sujeto genuino o básico no implica ni requiere asumir que la inherencia se cumpla sólo en el caso de los accidentes particulares para mantener consistentemente la particularidad de la dependencia de los accidentes. En última instancia, la dependencia de un accidente remite a un sujeto individual, pero el sujeto de un accidente puede considerarse también generalmente (Sócrates qua hombre), o también podemos hablar de la inherencia de una propiedad universal (color) en un sujeto individual (Sócrates) o en uno universal (hombre).

La distinción de la dependencia particular con respecto a la dependencia universal, propia de las especies y los géneros en su relación con los miembros individuales de la clase correspondiente, se mantiene intacta tras la última precisión concerniente a una ampliación de los sujetos de que dependen particularmente los accidentes. En efecto, con respecto a una propiedad accidental, no puede ser indiferente a qué sujeto se atribuye; su dependencia del sujeto individual determinado es parte del contenido conceptual y de las condiciones de existencia de un accidente. Así, aceptar la lectura que estoy proponiendo de la condición de inseparabilidad no nos obliga a analizar la inherencia, como sugería Ackrill, exclusivamente en términos de la inherencia de instancias de la propiedad accidental en individuos sustanciales. Los ejemplos aristotélicos de la inherencia en Cat. 2, 1a25-b3, se acomodan a la distinción de un sentido primario de ser-en, de acuerdo con el cual accidentes particulares inhieren en sustancias individuales, y otros sentidos derivados de esta relación, según los cuales los siguientes enunciados son explicables bajo el mismo esquema de la inherencia:

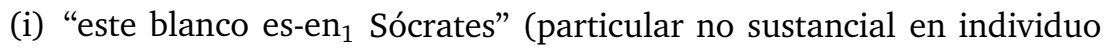
sustancial);

(ii) "el color es-en 2 Sócrates" (universal no sustancial en individuo sustancial);

${ }^{12}$ Cfr. Oehler 1997, p. 219. 
(iii) "el color es-en ${ }_{3}$ el hombre" (universal no sustancial en universal sustancial). ${ }^{13}$

Antes de abordar más directamente una reconstrucción del concepto de $P a$ quiero insistir un poco en la defensa de la interpretación propuesta de 1a24-25. Uno de los puntos principales de la propuesta de lectura que suscribo reside en que los accidentes particulares no son particulares meramente por el hecho de no predicarse de ninguna otra cosa. La indivisibilidad no explica completamente la unidad numérica de un particular no sustancial. La repetibilidad que caracteriza la identidad de las propiedades -el hecho de que es uno y el mismo color gris acero el de mi lámpara y el de mi reloj- pone en un mismo plano los accidentes universales y los particulares, pero ello mismo es un indicio de que no existe una relación entre identidad e individualidad en el caso de las propiedades no sustanciales. Así, la individualidad de las propiedades no puede explicarse recurriendo a su contenido conceptual, sino al número de su portador. Si la esencia de un accidente particular es indiferente con respecto a su repetibilidad, la definición de una propiedad no incluirá los posibles factores individualizantes que hacen ser particular a un accidente. ${ }^{14}$

A la diferenciación de distintos grados de inherencia que he propuesto se le puede dar sustento mediante una aclaración de Duerlinger, anticipada esquemáticamente por Allen, ${ }^{15}$ y que permite mantener una tesis aristotélica central de la metafísica de las Categorías: la prioridad de las sustancias individuales como genuinos sujetos básicos, sin que dicha tesis nos impida reconocer la función de sujetos que en sentido derivado asumen otra clase de entidades.

Hay, entonces, cuatro casos en que la relación de inherencia se realiza, tal como lo anticipaban $\mathrm{R} 2{ }_{1}-\mathrm{R} 22_{4}$; en ellos se puede reconocer una inherencia primaria y tres derivadas, así como un sujeto básico y tres funciones de sujeto derivadas, en cuanto que estos sujetos no son sujetos últimos, pues o bien son-en sustancias individuales, o bien se dicen-de tales sustancias.

$$
\begin{aligned}
& a \text { (este blanco particular } \\
& \text { determinado) es-en } x \\
& \text { (Sócrates) qua } S^{*} \text { si }
\end{aligned}
$$

(i) a no se dice-de ningún $S$, y

(ii) a cumple con las condiciones (i) y (ii) de (3);

\footnotetext{
${ }^{13}$ Cfr. Ackrill 1963, pp. 74 ss., p. 83; Lewis 1991, pp. 58 s.; Oehler 1997, pp. 219-227.

${ }^{14}$ Por ello, creo que la diferencia entre las propiedades no sustanciales particulares y generales que traza Owen - para quien sólo las segundas se predican de un sujeto y las primeras se presentan en múltiples sujetos- no alcanza a explicar la peculiaridad de los particulares no sustanciales. Cfr. Owen 1965, pp. 98-101. Vuelvo a tratar la propuesta oweniana aquí infra. Sobre las soluciones opuestas de Ackrill y Owen, cfr. Jones 1972.

${ }^{15}$ Cfr. Duerlinger 1970, pp. 185 ss.; Allen 1969, p. 35.
} 

$\left(3^{* *}\right) \quad A$ (color) es-en $1 x$ qua $S^{*}$ si
(i) $\quad A$ se dice-de $a$ qua $S^{* *}$, y
(ii) $a$ es-en 0 qua $S^{*}$;
$\left(3^{* * *}\right) \quad a$ es-en $2 F$ (hombre) qua $S^{* * *}$ si
(i) $a$ es-en $x$ qua $S^{*}$,
(ii) $F$ se dice-de $x$ qua $S$.
$\left(3^{* * * *}\right) \quad A$ es-en $F_{3}$ qua $S^{* * *}$ si
(i) $A$ se dice-de $a$ qua $S^{* *}$,
(ii) $a$ es-en $x$ qua $S^{*}$, y
(iii) $F$ se dice-de $x$ qua $S$.

El propósito de la esquematización precedente es mostrar, con anterioridad a la reconstrucción más detallada de la noción de accidente que emprenderé en las secciones siguientes, que articular tres relaciones derivadas y apoyadas en la inherencia primaria $\left(3^{*}\right)$, dando cabida en la relación de inherencia a las distintas entidades que admite el primer modelo ontológico de Aristóteles, resulta perfectamente coherente con la tesis de la prioridad ontológica de la sustancia individual o primera. Pero consideremos ahora el concepto mediante el cual es factible explicar los accidentes aristotélicos.

\section{Parte atributiva y sujetos}

En Cat. 5, 3a24-32, Aristóteles formula una aclaración colateral respecto del tema que viene desarrollando (explicación del género y la diferencia como partes de las sustancias, y aclaración de la diferencia específica como un componente de la sustancia que, si bien se dice-del sujeto, no se cuenta entre las sustancias segundas; cfr. $2 \mathrm{~b} 29 \mathrm{ss}.) .{ }^{16} \mathrm{El}$ estagirita se refiere ahora a las $P f$, las cuales son sustancias (3a30-31), siendo, además, en un sujeto como en un todo. Este recorte de las $P f$ explicita la tesis que Aristóteles introdujo anteriormente $(2,1 \mathrm{a} 24)$ con el objetivo de delimitar las $P a$. Las $\mathrm{Pa}$ son-en $S^{*}$ no qua partes en un todo, tesis que 5, 3a30 explica aclarando cuál es el sentido que le corresponde a 'parte' en el texto anterior. Así, obtenemos una delimitación, que incluye elementos semánticos, del modo en que un accidente no puede ser parte de una sustancia:

(4) $a$ es-en $x$ qua $S^{*}$ si $a$ no es una $P f$ de $x$ qua todo (cfr. 5, 3a31-32).

En efecto, sobre las $P f, 3 a 29-31$ (cfr. también 7, 8b15-21) permite establecer:

(5) $m$ es $P f$ de $x$ qua $S^{* * * *}$ si $m$ es una parte de $x$ qua todo.

\footnotetext{
${ }^{16}$ Sobre la ambigua posición de las diferencias específicas, cfr. Ackrill 1963, pp. 85 ss.; Oehler 1997, p. 266; Simplicio 1907, p. 98, 6-9.
} 
Lo que Aristóteles sostiene en 5, 3a29-32, es que las $P f$ son en un sujeto $\left(S^{* * * *}\right)$ únicamente qua partes de un todo. Así, una sustancia en la relación $P f R S^{* * * *}$ es un todo. A pesar de cierta vaguedad con que manejamos por ahora la noción de un todo físico, lo dicho nos permite entender más precisamente el tipo de sujeto que es $x$ en la mencionada relación $P f R S^{* * * *}$. Podemos aseverar, al menos, que $m$ no es-en $x$ qua $S^{*}$. Además, ya estamos en condiciones de demarcar aquí una relación de posesión en $P f R S^{* * * *}$, que es distinta de la inherencia que encontramos en la relación $P a R S^{*}$. Una $P f$ es parte de una sustancia en cuanto que el individuo de este tipo posee, por ejemplo, una mano o una cabeza como parte de su cuerpo (cfr. 15, 15b2). Si no estableciéramos esta distinción entre $P a$ y $P f$, nos veríamos obligados a sostener que las $P f$ no son sustancias; pero Aristóteles toma estas últimas partes como sustancias dentro de su primer modelo ontológico (7, 8a13-21, b15-21; también Metaph. V 8, 1017b10-13; VII 2, $1028 b 9-10 ; 16,1040 b 6-8)$. Las $P f$ deben no ser-en un sujeto para ser sustancias, tal como lo indica Categorías 5, 3a7-8, mediante el establecimiento del teorema según el cual es común a toda sustancia no ser-en un sujeto. En efecto, si las $P f$ son sustancias, como lo admite 3a29-31, no deben ser-en un sujeto ni constituyen propiedades inherentes o accidentes.

De (3) puede inferirse una ulterior aclaración acerca del estatus de $x$ qua $S^{*}$ de propiedades tipo $a$. He acentuado que lo que significa para $x$ ser un $S$ en R1 no es exactamente lo mismo que lo que significa para $x$ ser un $S^{*}$ en R2.

(6) En R1 se toma a $x$ como una instancia (individuo) de una especie (universal) definida por el enunciado que da el 'qué es' de $x$ en la relación " $F$ se dice-de $x$ "; luego $x$ es $S$ en R1 porque (i) $x$ está específicamente determinado por $F(2,1 \mathrm{a} 20-22)$, y (ii) $x$ no se dice-de ningún otro $S$ (1b3-6). ${ }^{17}$

(7) En R2, $x$ es $S^{*}$ no en virtud de ser definido por $a$ ( $x$ no constituye una instancia particular de aquello que se le atribuye $(a)$ en la relación

17 (6ii) es de importancia capital en la noción de sujeto individual último de Cat. - i.e. no meramente el sujeto individual representado por un accidente particular (tis grammatiké, tì leukón), que es sujeto de una determinación genérica que se dice-de él (e.g. ciencia, color); estos individuos no son sujetos últimos, en cuanto que tienen, a su vez y necesariamente, un sujeto $(S)$ en el que inhieren (Cat. 2, 1a25-28, 1b8-9)—. El registro lingüístico de esta posición fundamental de los sujetos se halla en el 'tís' que designa un individuo; pero el pronombre indefinido va siempre acompañado, en estos casos, del sortal especificador: tis ánthropos (cfr. e.g. Cat. 2, 1b4-5; 5, 2a13-14). El concepto del sujeto individual último ( $x$ ) está determinado, entonces, tanto por el carácter de la (i) indivisibilidad (átomon) (cfr. 2, 1b6-7) -i.e. por el hecho de que tales sujetos no son divisibles en otros sujetos (posteriores), al no decirse-de ninguna otra cosa-, como por el carácter de la (ii) independencia, pues existen separados de otra cosa. 
ser-en), sino que $x$ es $S^{*}$ de $a$ por el hecho de que propiedades tipo $a$ siempre y necesariamente son-en un sujeto. ${ }^{18}$

Con esto debe asumirse para las Categorías cierta marcada multivocidad en el significado de hypokeímenon, tal como he tratado de señalarlo mediante las aplicaciones de asteriscos a la función general del sujeto. Pero también debería notarse que los distintos tipos de sujetos se articulan sobre la base de la prioridad lógica y ontológica de un sujeto fundamental, función que en la metafísica de las Categorías es desempeñada únicamente por las sustancias individuales. En R2 también se reconoce una prioridad, como lo mostraba $\left(\left(3^{*}\right)-\left(3^{* * *}\right)\right)$, ya que allí el sujeto último que hacía posible las inherencias derivadas era $x$ qua $S^{*}$ en la relación $a R x$ (ser-en ${ }_{0}$ ). Pero distingamos, entonces, las dos diferentes funciones fundamentales que puede asumir un individuo sustancial en calidad de sujeto:

(8) (i) En R1, el hypokeímenon constituye una instancia particular determinada por un sortal en función especificadora e identificadora $(F)$;

(ii) $\quad x$ es $P c$ de $F$;

(iii) $x$ es necesariamente $F$;

(iv) $x$ es específicamente uno y el mismo $F$;

(v) $x$ es individual (numéricamente uno).

(9) (i) En R2, el hypokeímenon constituye el sujeto del cual a se enuncia sin definirlo, i.e. a no funciona como un sortal especificador ni individualizador con respecto a $x$;

(ii) $a$ es un atributo de $x$;

(iii) $x$ es contingentemente $a$;

(iv) $x$ puede admitir múltiples propiedades distintas $(a, b, c, \ldots)$ e, incluso, contrarias $(a \wedge \neg a)$ sin que ello produzca un cambio en su identidad ni en su unidad.

(9) no establece ninguna condición para que en R2 se admitan como $S^{*}$ únicamente individuos ( $c f r$. los pares $\mathrm{R} 22_{2}$ y R2 $2_{3}$ ).

A partir de aquí puede precisarse algo más la contingencia en R2: ${ }^{19}$

(i) Si $a$ es-en $x$ como en un $S^{*}$, entonces puede haber instancias $x_{i}$ para las cuales no vale $a$ es-en $x$, en cuanto ser- $x$ no presupone tener $a$;

\footnotetext{
${ }^{18}$ Creo que en las Categorías no se dice mucho más acerca de por qué determinadas entidades tienen (pueden tener) ciertos accidentes. Este hecho marca un límite más de la primera metafísica de Aristóteles.

${ }^{19}$ Moravcsik 1967, p. 89, subraya que esta condición está relacionada con la prioridad de la sustancia en el primer modelo ontológico de Aristóteles.
} 
(ii) dado que $x$ sigue siendo determinable como $S$ ( $x$ qua $F$ ) teniendo o no teniendo $a, a$ no determina la identidad ni la unidad específica de $x$; o sea, lo que es necesariamente $x$;

(iii) por tanto, las propiedades que son diferentes de las determinaciones que necesariamente pertenecen a $x$ son propiedades contingentes.

Categorías 3, 2a27-31, establece, además, la predicación del nombre de $a$ (' $a$ ') a $x$ en R2 como una explicación de la clase de predicación que tiene lugar en R2:

(11) Si $a$ es-en $x$ como en un $S^{*}$, entonces $a$ se predica nominalmente de $x$.

(11) precisa el tipo de relación que encontramos en $a$ es-en $x$. Esta relación puede denominarse atribución. La atribución (R2) se distingue de la predicación (R1) en que la definición del predicado gramatical se aplica al sujeto únicamente en la predicación, mientras que en la atribución, si bien ' $a$ ' se dice de $x$, las determinaciones esenciales del atributo (a) no se dicen-del sujeto. De esta manera, Aristóteles trata de explicar enunciados como "Sócrates es blanco", sosteniendo que blanco no se predica de Sócrates (5, 2a27-29). Aristóteles admite que en algunos casos sólo el nombre del atributo (' $a$ ') puede atribuirse al sujeto, pero de ninguna manera la definición de aquél (2a29-31); así, no hay margen para confundir esta forma de enunciación con la predicación estricta decir-de. ${ }^{20}$

Pero hay otra clase de sujeto y de relación atributiva que no debemos confundir ni con R2 ni con R1. La relación predicativa a la que ahora me refiero aparece en Cat. 6, 5a38-b10, aunque aquí voy a tomar en cuenta especialmente la formulación de la misma en los Segundos Analíticos (APo.) I 22, 82b37-83a35, donde Aristóteles distingue sujeto gramatical de sujeto lógico (83a5-7), recurriendo a que, en enunciados como "lo blanco es madera", el sujeto gramatical no designa aquello en virtud de cuya característica propia se predica lo que se dice de él en el lugar del predicado.

\footnotetext{
${ }^{20}$ Aristóteles es impreciso en el uso de kategoreîn aquí (Cat. 5, 2a30) cuando afirma que nada impide que en algunos casos el nombre (del atributo) se predique del sujeto. Con esta concesión, Aristóteles pretende hacer lugar a la relación según la cual un blanco (particular) es-en un cuerpo (particular) (2a31-34). Él piensa que es plausible considerar que el nombre se predica del sujeto por el hecho de que decimos cosas tales como "este cuerpo es blanco" y podemos hacer referencia al sujeto $\left(S^{*}\right)$ designándolo, en tales casos, como "el blanco". Aristóteles cree que la predicación nominal puede explicar el hecho de que se dice del cuerpo que es blanco (2a32), sin hacernos suponer erróneamente que esa atribución nos autoriza a predicar del sujeto las propiedades que definen al predicado. La predicación nominal aclara la similitud en la forma gramatical y la desemejanza en la forma lógica que existe entre enunciados como "Sócrates es hombre" y "Sócrates es blanco".
} 
Esta aclaración le permite a Aristóteles explicar (83a7-9) que la relación entre el sujeto ${ }^{21}$ y el predicado gramaticales es accidental (cfr. hôi symbébeke leukôi eînai xýlon, 83a5-6), donde "a es accidentalmente b" se opone a " $x$ es por sí mismo $F$ " (i.e. " $x$ qua $S$ es $F$ ").

Esta distinción no debe confundirse, tal como Aristóteles lo subraya allí mismo (83a9-14), con una diferenciación entre enunciados donde se predica del sujeto un accidente, sin que el sujeto gramatical sea accidentalmente sujeto (o sea, enunciados de la forma " $x$ tiene $a / A$ ", donde $x$ funciona como $S^{*}$ ). En enunciados donde el sujeto gramatical coincide con el sujeto lógico, del sujeto pueden decirse predicados esenciales ("Sócrates es hombre") o accidentales ("Sócrates es culto", "la madera es blanca"). En resumen, una cosa es (i) introducir en un enunciado una propiedad accidental como sujeto gramatical (a es accidentalmente sujeto) y otra es (ii) admitir que $x$ sea sujeto genuino (lógico) de una propiedad accidental con la cual se halla en una relación no fundada en la propia característica definitoria del sujeto.

En el enunciado "lo blanco es madera" no es en virtud de la cualidad de blanco que algo es una madera. En efecto, debe decirse que algo blanco es accidentalmente de madera, por cuanto, como sujeto de la determinación madera, esa cosa blanca no tiene tal característica (ser de madera) en virtud de ser blanca. Entonces, puesto que 'lo blanco' no es el sujeto lógico del predicado ('madera'), puede explicarse que algo blanco sea accidentalmente de madera. Si antes he distinguido, siguiendo la jerga de Alan Code, entre ser y tener para R1 y R2, ahora debería introducir una ulterior categorización para este 'es' accidental, donde una propiedad contingente es accidentalmente sujeto $\left(S^{* * * * *}\right)$. Podemos identificar esta relación predicativa mediante la notación acontecer:

(12) $a$ es accidentalmente sujeto de $b$ si le acontece a $a$ ser $S^{* * * * *}$ de $b$.

Y deberíamos clarificar la relación acontecer sosteniendo que
(13) a $a$ le acontece $\operatorname{ser} S^{* * * * *} \operatorname{de} b$ si
(i) $a$ es $A$ y $b$ es $B$ (donde $A \neq B$ )
(ii) $x$ tiene $a$ y $x$ tiene $b$;
(iii) $b$ se dice de $a$ sólo en virtud de (ii).

Sobre esta base puede entenderse que a continuación (83a14-35) Aristóteles estipule que kategoreîn se da propiamente en el caso en que coinciden el sujeto gramatical y el lógico (" $x$ es (es) $F$ ", " $x$ es (tiene) $a$ "); en esos casos encontramos, entonces, una predicación sin más (kategoreîn haplôs,

\footnotetext{
${ }^{21}$ Para distinguirlo de los restantes sujetos deberíamos designar el sujeto gramatical con la notación $S^{* * * * *}$.
} 
83a16). En cambio, una predicación de la forma "a es b" es una predicación accidental o es accidentalmente una predicación (katà symbebekòs dè kategoreîn, 83a16-17), es decir, no es una genuina predicación, sino un caso en el cual tanto el sujeto como el predicado no son sujeto y predicado por la relación que guardan entre sí. En la predicación accidental, el predicado califica sólo de una manera aparente o incidental al sujeto gramatical. A esta determinación aparente se opone una determinación genuina, cuyas variantes son una predicación esencial y otra accidental. Aristóteles restringe al ámbito de las dos clases de predicación genuina las proposiciones que entran en las demostraciones (83a20-21) y sostiene que, en tales casos (cfr. 83a21-22, 24-25), una cosa se predica de otra (hèn kath' henòs kategorethêi, 83a22-23, b17-18). ${ }^{22}$ En resumen:

(14) $a$ es un sujeto accidental si $b$ se predica accidentalmente de $a$.

Volviendo a la consideración de las dos principales formas predicativas que constituyen la base de la teoría de las Categorías y en correspondencia con el doble sentido de 'hypokeímenon' en esas dos relaciones predicativas (R1 y R2), cabe establecer, al menos, una distinción en la noción de 'individuo' entre $x$ en R1 y a (tis grammatiké, tì leukón) en algunas de las R2. La individualidad de $x$ se explica en términos de indivisibilidad; en cambio, la de algunos $a$ no debe poder explicarse en los mismos términos si es el caso que $\mathrm{R} 2$ no es equiparable a R1. Tanto $\mathrm{R} 2{ }_{1}$ como $\mathrm{R} 2{ }_{4}$ no establecen una predicación especificadora que permita tomar a $x$ como un individuo (numéricamente indivisible) en cuanto instancia de $F$. Sin embargo, los individuos no sustanciales -que distinguiré mediante la notación $a^{*}$ para destacar que no se hace mención meramente a una propiedad indivisible, sino además numéricamente una- se definen en cuanto individuos, por un lado, como instancias particulares de un universal (cfr. Cat. 2, 1b1-2), es decir, comparten un rasgo de la individualidad que se atribuyó a los individuos sustanciales, tal es: la indivisibilidad. En efecto, Aristóteles se refiere ( $c f r .2,1 b 6-7$ ) conjuntamente a los individuos sustanciales y no sustanciales cuando especifica la condición de indivisibilidad (a través del requisito de no-predicación) que le corresponde a ambas clases de entidades ya distinguidas más arriba. Pero Aristóteles sostiene que hay individuos en sentido fuerte o estricto, y que tales son los individuos sustanciales. Un indicio lingüístico de esto se encuentra en el uso de haplôs en 1b6,

\footnotetext{
${ }^{22}$ En la literatura aristotélica se ha denominado frecuentemente a las dos clases de predicación introducidas en APo. I 22 con los apelativos de "predicación natural" y "predicación no natural" o "antinatural". Sobre el lugar sistemático que ocupa el tratamiento de las mismas dentro del problema de la finitud de las premisas, el carácter demostrativo de la ciencia — que se plantea entre I 19-23-y los predicados convertibles, así como también para una discusión detallada que incluye una síntesis informativa de las principales opiniones de los intérpretes, cfr. Detel 1993, II, pp. 375-400.
} 
pues frente a ese sentido estricto de 'individuo', que demarca entidades que no sólo no se dicen-de, sino que, además, tampoco son-en -o sea, cosas que son sujetos genuinos y básicos-, Aristóteles parece sugerir, a continuación (1b7-8) —-mediante una proposición adversativa referida a lo que sostiene el enunciado precedido por haplôs - que hay cosas que pueden ser individuales incluso siendo inherentes en otras entidades. ${ }^{23}$ Me parece suficientemente tangible que Aristóteles está tratando aquí de demarcar una clase diferente de individuos, los individuos accidentales, dentro de las entidades individuales. Análogamente, debería ser claro que esta segunda clase no cumple con uno de los dos requisitos con que sí cumple la primera (ya que $x$ no sólo no se dice-de ninguna otra cosa como de un sujeto, sino que tampoco es-en otra cosa como en un sujeto, $c f r$. 1b3-6), pues los $a^{*}$ son individuos a pesar de ser-en.

Esta última tesis presenta algunas dificultades en cuanto a la interpretación del texto, pues el "nada impide" aristotélico (1b8), que califica con una especie de advertencia y concesión en este contexto argumentativo la individualidad de las entidades no sustanciales, no explica con claridad los siguientes hechos:

(i) si el que algunas entidades no sustanciales sean individuales constituye un rasgo que ellas poseen a pesar de ser inherentes - como lo he sugerido al final del párrafo anterior-, o

(ii) si ellas son individuales prescindiendo del hecho de que son inherentes.

Pero también, apelando a ciertas intuiciones plausibles, podría conjeturarse - y en la extensa discusión crítica acerca de los individuos no sustanciales se lo ha hecho- una tercera variante - para la que, empero, este pasaje no ofrece mayor sustento-, según la cual

(iii) la individualidad característica de los particulares no sustanciales reside no sólo en su indivisibilidad específica, sino en el hecho de que son propiedades indivisibles que pertenecen a individuos sustanciales según el modo de la inherencia. Así, tales propiedades indivisibles serían particulares sólo en virtud de ser inherentes en individuos sustanciales.

Esta cuestión parece difícilmente resoluble apelando al escueto texto aristotélico. ${ }^{24}$ En este escrito no voy a discutir expresamente, como ya señalé, el concepto de individuo y la cuestión de los accidentes particulares dentro

${ }^{23}$ Cat. 2, 1b6-8: "las cosas indivisibles y absolutamente unas según el número no se dicen de ningún sujeto, pero nada impide que algunas sean en un sujeto" (haplôs dè tà átoma kaì hèn arithmôi kat' oudenòs hypokeiménou légetai, en hypokeiménoi dè énia oudèn kolýei eînai).

${ }^{24}$ Para una discusión relacionada con este tema cfr. Strawson 1953/1954, pp. 56 s. 
de las Categorías. Pero mi tesis se inclina a explicar la opción (i) en términos de (iii), o sea, desfavorezco la inseparabilidad débil que sustentaría la opción (ii), ya que, de otra manera, Aristóteles se vería privado de herramientas para distinguir entre la especie indivisible del color gris acero y el gris acero particular de mi reloj, por un lado, y el gris acero de mi reloj y el gris acero idéntico de mi auto, por el otro, como colores numéricamente diferentes de la misma especie cromática.

Dejando de lado ahora este obstáculo, y para dar un cierre provisional a este tópico antes de volver a abordarlo al final de este artículo, quiero subrayar que los particulares no sustanciales presentan un rasgo distintivo elemental frente a las sustancias en general, ya que los particulares no sustanciales son-en sustancias individuales (cfr. 2, 1a25-26, a27-28). El punto relevante para determinar el carácter de la individualidad de lo que creo constituye, para Aristóteles, una genuina segunda clase de individuos - los cuales, empero, y por el mismo hecho de su inherencia recién subrayado, no pueden ser los sujetos básicos de esta metafísica- reside en el hecho de que los particulares no sustanciales existen como instancias específicamente determinadas de manera completa necesariamente en otros individuos, mientras que en el caso de las sustancias individuales su propia determinación completa va acompañada de su no-inherencia. La tesis de Aristóteles es que los individuos que constituyen el pilar fundamental de su ontología son últimos o básicos tanto porque son específicamente indivisibles, como por el hecho de que no dependen de otro sujeto para existir.

Pero los accidentes conjugan en su propia explicación dentro del modelo de las Categorías una inserción en la teoría de la predicación y en la distinción semántica introducida en el capítulo primero. Veamos, entonces, cuál es la fundamentación completa de los accidentes aristotélicos.

\section{Predicados, parónimos y accidentes}

Russell Dancy ${ }^{25}$ abordó la distinción aristotélica entre enunciados donde (i) se predica la especie del sujeto en cuestión, y otros donde (ii) se predica del mismo sujeto algo meramente accidental, tratando de aclarar la diferencia entre el predicado de "Sócrates es gramático" (Cat. 5, 3a4-5) y el sujeto de "la gramática es un (una especie de) conocimiento" $(2,1 \mathrm{~b} 2-$ 3). Equiparar el predicado del primer enunciado al sujeto del segundo arroja la absurda proposición "Sócrates es (un) (una especie de) conocimiento". Dancy llama la atención sobre 5, 2b29-3a6 (cfr. 3a3-5), donde Aristóteles afirma que ítems como 'corre', 'blanco', 'gramático' y otros del mismo tipo se predican (kategoreîsthai, 3a4) de las sustancias primeras y segundas - de estas últimas se predican aquellos ítems no sustanciales, en

${ }^{25}$ Cfr. Dancy 1975, pp. 349 ss.

Diánoia, vol. L, no. 55 (noviembre 2005). 
tanto que las sustancias segundas se predican de las primeras mostrando (delô̂, 2b31) las sustancias primeras, como tales (2b29-31)-. Este pasaje se propone distinguir -apoyándose en otros anteriores con el objetivo de explicar la diferenciación que allí se introduce- una predicación esencial (apodidôi tí estin, 2b32) de otra accidental (allotríos éstai apodedokós, 2b35) para establecer un criterio — aplicable a la predicación esencialque nos permita demarcar cuáles ítems son sustanciales aparte de las sustancias individuales (cfr. 2b29-30). Así, la noción de kategoreîtai o légetai no puede ser la misma cuando relaciona, en un caso, una propiedad esencial con un sujeto, y cuando, en otro caso, relaciona una propiedad accidental con un sujeto.

Para dar cuenta de que tanto 'hombre' como 'blanco' se predican de Sócrates, a pesar de la diferencia lógica irrebasable que existe entre ambos predicados, Dancy cree estar obligado a asumir que 'predicado de' no significa lo mismo que 'dicho de' —lo que es correcto—, y, además, que 'predicado de' "debe ser una relación más general que incorpora tanto a 'ser dicho de' cuanto a 'ser en'". ${ }^{26}$ Esta última conclusión no se sostiene a partir del texto comentado por el autor ni a partir de otros de las Categorías. Me inclino a pensar que Aristóteles asume légetai con un carácter casi no terminológico y, en cambio, usa kategoreîtai, en ocasiones, con una carga terminológica, la cual, empero, no siempre alcanza para distinguir R1 de $\mathrm{R} 2$. Al respecto, es ilustrativo el pasaje de 3, 1b10-12, que usa ambos verbos para explicar la transitividad en la aplicación de un predicado. En 5, 2a27-34, Aristóteles usa kategoreîtai y kategoreîsthai para sostener que de las cosas que son-en un sujeto la definición no se predica del sujeto; además, se sirve del mismo verbo para decir que el nombre sí puede predicarse del sujeto. En 2a32 usa légetai para sostener que un color -que es-en un sujeto- se dice del sujeto; esto constituye una afirmación parentética llamada a explicar algo que ya vimos: sólo el nombre se predica ( $c f r$. 2a31-32, kategoreîtai) del sujeto, sin que la definición de la cosa nombrada se predique del mismo (kategorethésetai, 2a33-34). En cualquier caso, me parece evidente que Aristóteles admite y trata de explicar que 'gramático' se dice/predica de Sócrates, sin que esto implique que dicha relación deba subsumirse bajo R1. En R2 encontramos, entonces, una predicación nominal.

En relación con esto último, Dancy sostiene ${ }^{27}$ que Aristóteles toma como una prueba de la corrección de la definición el hecho de que el enunciado que da la definición de algo pueda sustituir siempre salva veritate al nombre de la cosa (cfr. Top. VI 1, 139a25-27, a36-b3; 5, 142b30-35; VII 5, 154a34b1). Esto indica que el estagirita no admite que si $F$ se dice de $x$ en el

${ }^{26}$ Cfr. Dancy 1975, p. 353.

${ }^{27}$ Cfr. Dancy 1975, p. 355.

Diánoia, vol. L, no. 55 (noviembre 2005). 
sentido de R1, la definición de $F$ no se predique de $x$. Por ello, Aristóteles no pudo estar admitiendo en las Categorías que "Sócrates es gramático" representa una predicación en el sentido de R1.

La teoría de los accidentes se elabora en las Categorías recurriendo a la noción semántica de paronimia, así como en la otra relación fundamental - R1- el ítem predicado, o sea, la esencia o un componente esencial de algo, se aclara apelando a la sinonimia. ${ }^{28}$ La noción de paronimia se introduce en 1, 1a12-15. Una formulación de la paronimia puede ser la siguiente:

(15) a es parónimo si (i) a obtiene su denominación (' $a$ ') a partir de algo $A$, de cuyo nombre (' $A$ ') difiere en la forma gramatical (en la desinencia) ya que ' $a$ ' es una forma adjetivada que se deriva del sustantivo ' $A$ ';

(ii) pero la definición de un parónimo es la misma que la del nombre correspondiente a su entidad de origen.

Destaquemos ahora algunos aspectos generales de la paronimia. Como lo expresa (15), un parónimo implica una dependencia con respecto a la entidad de la que obtiene su denominación (apó tinos prágmatos). Esa dependencia nominal refleja la dependencia existente entre los ítems designados por el parónimo y el sustantivo abstracto. La paronimia da cuenta de una vinculación no de dos cosas con un nombre y su definición, como se da en los extremos representados por la sinonimia y la homonimia, pues la paronimia está constituida por una única relación de derivación entre dos cosas expresadas por sus respectivos nombres. En cambio, tenemos un caso de homónimos cuando dos cosas diferentes en cuanto a su definición guardan sendas relaciones diversas con un nombre. Dos cosas son sinónimas, por otro lado, cuando cada una de ellas guarda una misma relación con la estructura (ousía) que define a ambas. ${ }^{29}$ Pero parónima se dice primero la entidad derivada y luego su propio nombre. Entonces, la homonimia y la sinonimia implican, respectivamente, un significado diferente y un mismo significado asociado a los términos involucrados en tales relaciones; en

\footnotetext{
${ }^{28}$ Sobre la semántica de Cat. 1 cfr. Oehler 1997, pp. 189-212; Ackrill 1963, pp. 71-73. La explicación de las nociones semánticas aristotélicas en términos de relación, que propone Oehler, me parece especialmente clara. La paronimia no asume en Cat. 1 ni en 8, 10a32 ss. la función de una herramienta para elaborar la teoría de la unidad focal del significado de 'ser', una función que es característica de la metafísica madura (cfr. Oehler 1997, pp. 197 ss.). Sin embargo, dicha teoría constituye una elaboración a partir de la paronimia presentada en Cat. —así como de nociones semánticas introducidas en textos como Tópicos (Top.) II 3, 110b16—, algo que es coherente con la explicación del accidente mediante aquella noción.

${ }^{29}$ Se asume aquí que la definición es la fórmula lingüística de la esencia (Top. I 5, 101b38).
} 
la paronimia, el significado del parónimo proviene del término de origen. Esto debería servir para afirmar que la paronimia no constituye una categoría gramatical, sino semántica, en cuanto que —asemejándose en esto a las otras dos nociones semánticas- ella establece una relación entre entidades sobre la base lógica del significado, aun cuando es cierto que la derivación nominal que caracteriza a los parónimos (paterno $<$ padre) responde a un esquema gramatical.

Aristóteles se sirve de los parónimos a fin de aclarar a qué categoría pertenece cierta entidad, es decir, qué significado categorial corresponde a un adjetivo derivado; y halla que tanto éste como lo designado por el nombre abstracto de origen deben subordinarse bajo la misma categoría o clase de entidades. Por ejemplo, el gramático y la gramática son ambos cualidades.

Como lo señaló Simplicio, ${ }^{30}$ la paronimia es, a diferencia de la sinonimia y la homonimia, una relación asimétrica; o sea, si graficamos con $P$ la relación parónima, vale que si $a P b$, entonces $\neg b P a$.

Los dos últimos comentadores de las Categorías coinciden básicamente en una opinión bastante difundida, según la cual la paronimia no desempeña un papel relevante en la primera teoría metafísica de Aristóteles. ${ }^{31}$ Pero, contrariamente a esto, la noción del parónimo le permite a Aristóteles abordar explicativamente los accidentes. 'El blanco' designa el sujeto que tiene la propiedad cuyo nombre es 'blancura', de la cual el adjetivo 'blanco' se deriva; una cosa se denomina 'blanca' en virtud del hecho de ser portadora de esa propiedad. Pero la definición del adjetivo 'blanco', que es la misma que la de 'blancura', no se aplica al sujeto en cuanto portador de esa propiedad. Aristóteles intenta explicar esta relación entre el sujeto y la clase especial de propiedades a la cual pertenece 'blanco' recurriendo a lo que llamo aquí predicación nominal, que intenta clarificar la noción de accidente. La primera metafísica de Aristóteles permanece ajena a otra serie de problemas que se avizoran ya con este planteamiento de la paronimia. Me refiero a la cuestión de la clase de entidad que es un sujeto tomado en conjunto con su accidente; una entidad a la que no cabe aplicar la definición del accidente. Toda la problemática de los compuestos accidentales resulta en gran medida ajena al tratamiento de las sustancias y los

${ }^{30}$ Cfr. Simplicio 1907, p. 37, 34.

${ }^{31}$ Cfr. Ackrill 1963, p. 72; Oehler 1997, p. 209; y también Wedin 2000, pp. 18-20. Para Wedin (p. 20), la paronimia sólo provee casos típicos de propiedades inherentes, pero no puede desempeñar un papel sistemático en la explicación de ellas por el hecho de que no habría un solapamiento entre casos de inherencia y de paronimia (e.g. el nombre que se aplica en griego al hombre virtuoso (spoudaíos) no se deriva gramaticalmente del nombre de la propiedad correspondiente, la virtud (he areté )). Más allá de que se acepte o no que tales ejemplos representan instancias de lo que Montgomery Furth llamó "paronimia degenerada", Wedin rechaza de plano que esta semántica sirva más que para sólo clasificar un grupo de ítems diferentes de los sinónimos y los homónimos. 
accidentes que se hace en las Categorías. Sin embargo, es interesante que un aspecto central de los compuestos accidentales, el de su definición e identidad, se suscita por la denominación parónima que cabe a un sujeto en cuanto portador de un accidente (cfr. 8, 10a30-32), pues el compuesto accidental 'el blanco' incorpora una combinación de un sujeto y un accidente sin que su propia identidad se equipare a ninguno de sus componentes y sin que pueda definirse esa clase de compuesto prescindiendo del añadido de nociones que no guardan entre sí cierta relación de inclusión. Con esto último hago alusión a esa clase de relación según la cual la entidad definida no incluye cosas diferentes en su propio enunciado. Tal es parte de la cuestión que plantea el enunciado de los compuestos accidentales.

Así, puede decirse que las cuestiones abiertas son dos: en primer lugar, si los compuestos accidentales representan entidades reconocibles con una unidad distinta de sus partes - lo que lleva a la necesidad de precisar qué puede querer decir allí 'ser distinto de' y 'ser idéntico a', así como a explicar la unidad de las entidades-; en segundo lugar, ¿qué clase de identidad podría corresponderle a esos compuestos en los cuales el sustantivo de la propiedad de origen ('blancura'), el adjetivo que nombra la propiedad inherente ('blanco') y el sujeto llamado según su función de portar esa propiedad ('el blanco') no tienen el mismo significado, pero introducen una y la misma entidad? Tales son planteamientos contenidos en la teoría aristotélica de los accidentes y en el papel metafísico que desempeña la paronimia, pero que Aristóteles suscita recién en Metaph. VII 4-6, cuando examina el vínculo que mantienen las entidades sustanciales con la esencia y la definición, a partir de lo cual se genera la necesidad de clarificar de qué cosas hay definición con el objetivo de seleccionar un ámbito de entidades primarias y sustanciales. Es evidente que tales planteamientos resultan ajenos a la posición de las Categorías, donde la definición se asume poco menos que como resultado de un procedimiento incontrovertido. Es por esto que Aristóteles acepta allí que hay definiciones por igual de dos clases de entidades que él distingue netamente en su ontología: sustancias y accidentes. Su noción de definición en el contexto de las Categorías constituye un sobreentendido. Sólo cuando Aristóteles observa que la definibilidad es un criterio y una condición de las sustancias - un criterio asociado a la unidad metafísica de una cosa- llega a suscitar el problema de la definición de los compuestos accidentales y de los accidentes.

Como vimos, los sustantivos de que se derivan los parónimos son nombres de propiedad ('justicia', 'belleza', 'sabiduría', 'rojez', 'altura', etc.). Dancy $^{32}$ ha insistido acertadamente en que este análisis que vincula los parónimos al registro lingüístico de la modificación en la terminación del sustantivo puede aplicarse también a casos como el de leukón (blanco),

${ }^{32}$ Cfr. Dancy 1975, p. 361. 
donde, en las expresiones lingüísticas que tiene en cuenta Aristóteles, ${ }^{33}$ no se registra una diferencia lingüística entre cualidad y calificado. ${ }^{34}$ Lo relevante ahora es que el aspecto semántico del parónimo explica que entidades cuyos nombres son parónimos están caracterizadas por una doble dependencia. La primera dependencia se expresa en el nombre de la entidad de proveniencia del parónimo correspondiente ('blancura', en el caso de 'blanco'); la segunda dependencia se expresa en la inflexión gramatical a que se somete el sustantivo abstracto ('blancura') para que sólo entonces represente el nombre de un parónimo ('blanco'), de la propiedad de un sujeto. Así, el parónimo guarda una doble relación de dependencia: (i) con respecto a la propiedad abstracta y (ii) con respecto al sujeto portador de la propiedad.

Parte de la intuición que aquí está en juego consiste en que las propiedades abstractas accidentales sólo pueden mantener una relación con el sujeto en el que existen mediante una especificación; por ésta, las propiedades abstractas se convierten en algo determinado que sólo en virtud de esa modificación — registrada en la inflexión característica del parónimopuede existir en un sujeto; es decir, existir sin más, ya que todo lo que existe y no es un sujeto genuino o una sustancia primera o bien es materialmente en un sujeto, o bien se dice-de un sujeto, o bien es-en un sujeto. Esta modificación es obligatoria para la existencia de las propiedades abstractas accidentales, ya que ellas no existen por sí mismas - como lo establece Aristóteles en general para todo aquello que en su existencia depende de un sujeto (cfr. Cat. 5, 2a34-35) - ${ }^{35}$ ni, a diferencia de las propiedades abstractas sustanciales, dan la determinación de lo que algo (un sujeto o sustancia primera) es. Esta última función de las sustancias segundas ex-

\footnotetext{
${ }^{33}$ Pues existe en griego el sustantivo abstracto leukótes, y ya Platón había implementado en el Teeteto (Tht.) el recurso lingüístico de la expresión asociada al nombre correspondiente del adjetivo (blancura $<$ blanco, dureza $<$ duro, en general, cualidad (poiótes) $<$ calificado o quale (poión)) para referirse a la propiedad del caso.

34 Textos como Cat. 7, 6b11-14, permiten afirmar que la condición de la paronimia no rige sólo para lo que Aristóteles toma estrictamente como cualidades.

35 Moravcsik 1967, pp. 83 s., destaca que, según Ackrill, los términos generales (e.g. 'sabio') y el término singular abstracto correlacionado ('sabiduría') introducen, en opinión de Aristóteles, la misma entidad. No creo que constituya una dificultad para esta interpretación el hecho de que 'sabio' introduce un compuesto de la propiedad particular del sujeto y de este último (Sócrates-sabio), ya que la tesis metafísica de Cat., en este aspecto, es que las propiedades (tanto esenciales como accidentales) no existen separadas, por lo cual toda introducción justificada de propiedades requiere asumir en ellas alguna modificación proveniente del hecho de que dependen de un sujeto. La tesis antiplatónica al respecto dice que no existe la sabiduría, sino que sólo existen hombres sabios. Jones 1972, pp. 121 s., atribuye a Aristóteles dos usos de formas sustantivadas como 'lo blanco': uno por el que se designan tipos de matiz de la propiedad, y otro mediante el que se denotan objetos blancos. Obviamente, el objeto y la propiedad no admiten la misma definición, y el segundo uso corresponde a lo que Aristóteles denominará "cosas dichas accidentalmente" (cfr. Metaph. VII 6, 1031b22-25), es decir, cosas cuya unidad (entre el sujeto y el accidente) es meramente accidental.
} 
plica que su nombre no registre inflexión, ya que la característica abstracta que expresa su propio significado (e.g. el significado del nombre 'hombre') constituye el rasgo peculiar con que Aristóteles entiende la determinación de los individuos sustanciales dentro de su primer modelo ontológico. Ése es el rasgo que asegura la sinonimia estricta existente entre Sócrates y hombre.

Por otro lado, dicho carácter de las sustancias segundas arroja como consecuencia que (i) Aristóteles toma la determinación esencial (la determinación que informa qué es $x$ ) de los individuos sustanciales como idéntica a la clase de determinación esencial correspondiente a entidades sustanciales abstractas - es decir, dos entidades de distinto tipo (primeras y segundas, en la terminología aristotélica) poseen una misma clase de determinación esencial, algo que no puede pasar por una tesis evidente-, y que (ii) la función determinativa que están llamadas a desempeñar las sustancias segundas esté asumida ya en la misma determinación bajo la cual se entiende $x$ qua específicamente determinado bajo el sortal ' $F$ '. (ii) pretende expresar que lo que es $x$, siendo ello mismo parte de $x$ (una parte constitutiva de $x$ ) -pues $x$ se toma siempre ya como algo (ti) determinado (tóde)—, representa una determinación que ya está dada en la aprehensión de $x$ como $F(x)$, ya que $x$ resulta siempre ya aprehendido bajo la determinación de un sortal en función identificadora. ${ }^{36}$

Retomando una buena idea de Barrington Jones, podríamos decir que lo que aporta la metafísica madura en este aspecto es una explicación relativa a las condiciones bajo las cuales puede contarse como una unidad (esto es un $F$ ) cierta masa material dotada, entre otras, de la característica de la persistencia, de tal manera que mediante esa operación estamos en condiciones de identificar una cosa como (un) algo (ti) individual de tal clase (tóde). Tales son cuestiones que el primer modelo ontológico aristotélico no fundamenta, pero con las que tiende a operar al usar términos sortales (e.g. 'hombre') para individualizar objetos a los que toma ya como individuos. Este problema concerniente a la individuación en relación con términos sortales o generales ('hombre', 'humanidad') se plantea para el caso de sortales usados en función identificadora; no, en cambio, en el de otros términos generales (e.g. 'cultura'), cuya función no es identificar algo, sino describir características de un objeto que no forman parte de su esencia ni determinan su identidad. Tales sortales llegan a individualizar ciertas características de algo mediante su inflexión parónima, por la cual se describe, por ejemplo, el carácter de culto de Sócrates. Pero, en última instancia, como sostengo en este trabajo, ${ }^{37}$ la individualidad de ese carácter depende de la individualidad de su portador. Como consecuencia

\footnotetext{
${ }^{36}$ Ésta es la razón por la que Jones 1975, pp. 170 y 167, sostiene que en las Categorías se impulsan los ítems como ser un hombre a un "limbo ontológico".

${ }^{37}$ Más adelante extraigo algunas consecuencias de esta tesis; véase infra § 4.
} 
de ello, el aspecto individual de una propiedad tiene pleno sentido y contenido sólo en cuanto dicha propiedad depende de la individualidad del sujeto. Así, la individualidad del carácter de culto perteneciente a Sócrates depende de ciertos rasgos individuales de su portador, rasgos que no se repiten necesariamente en otros portadores individuales a los que calificamos igualmente de cultos. Si el sujeto es individual, la cualidad particular existente no puede ser sino individual, en cuanto que sólo existe en individuos, y su propia determinación (su contabilidad) únicamente se alcanza a partir de su relación con la individualidad de cada uno de sus portadores particulares. Esta última condición, obviamente, no se da en el caso de sustancias abstractas que, por definición, se dicen de una multiplicidad de individuos y no dependen de los rasgos individuales de ninguno de ellos en particular, ni en su propia existencia ni en el contenido semántico de su propia determinación.

La determinación y la definibilidad de las sustancias individuales no son un axioma del esencialismo aristotélico, que comienza a desarrollarse articulando las condiciones de esa "unidad sellada" que constituye, en el primer modelo ontológico, la estructura esencial $F(x)$. En efecto, ésta es una estructura susceptible de ser fundamentada, tal como lo demuestra el avance que, en ese sentido, realiza el segundo por sobre el primer modelo ontológico de Aristóteles, cuando el estagirita intenta explicar cuáles son las condiciones que un sujeto debe satisfacer para admitir una determinación esencial o para ser estrictamente identificable e individualizable, y lo hace apelando al desarrollo del modelo materia-forma, que sirve para dar cuenta de la tesis esencialista fundamental concerniente al rechazo de los meros particulares. Mediante dicha teoría se explica la estructura de lo que son las sustancias primeras de las Categorías.

El uso de infinitivos en 7, 6b11-14 (estar en decúbito, estar erguido, estar sentado) para designar parónimos plantea algunas dificultades. Estoy de acuerdo con Dancy en la explicación que da acerca del sentido ontológico que tiene ese uso de infinitivos. ${ }^{38}$ Probablemente, Aristóteles usa allí infinitivos con el objetivo de distinguir entidades abstractas (universales sustanciales y no sustanciales) o independientes (individuos sustanciales) respecto de la clase de entidades que no comparte ninguna de esas dos características, es decir, los particulares no sustanciales. El mismo pasaje deja en claro que Aristóteles cree adecuado explicar los accidentes particulares mediante la paronimia. Allí utiliza, entonces, el recurso de los infinitivos,

\footnotetext{
${ }^{38}$ Para sostener esta interpretación, Dancy 1975, p. 361, n. 34, remite a Top. II 2, 109b4; Cat. 15, 15b27-30, y advierte que ese uso de infinitivos para designar propiedades que no existen separadas - parónimas - difiere del que Aristóteles da al mismo recurso lingüístico en Metaph. VII 1, 1028a20-21, con el objetivo de denotar propiedades tomadas como (si existieran) separadas. Pero ello no implica que las tesis filosóficas generales de ambos pasajes disientan una de otra.
} 
entendiendo esta forma verbal no como intercambiable con los parónimos, sino como denominadores de un tipo de accidentes (las posiciones o théseis) que se representan gramaticalmente de manera adecuada mediante infinitivos, en la medida en que éstos designan acciones que sólo existen en cuanto son ejecutadas por un sujeto. Los infinitivos parecen encarnar la intención de demarcar entidades que dependen del sujeto y son concretas o particulares. Este último rasgo es lo que le da sentido a la apelación a la paronimia que allí hace Aristóteles, pues los sustantivos que introdujo antes (el decúbito (he anáklisis), el estar erguido (he stásis) y el estar sentado (he kathédra)) designan entidades que Aristóteles ya ha clasificado como dependientes. En efecto, como posiciones, todas se dicen en relación con algo (6b12). Pero la relatividad que caracteriza a los infinitivos que designan parónimos no es meramente la que corresponde a los sustantivos abstractos asociados, como lo señala la contraposición que Aristóteles establece al declarar que los infinitivos, como tales, no designan posiciones, sino que nombran algo que obtiene la denominación del caso a partir de los nombres de posición respectivos. Los infinitivos (tò anakeklísthai, hestánai, kathêsthai) y su explicación como parónimos, en cuanto derivados de los sustantivos respectivos (paronýmos dè apò tôn eireménon théseon légetai, 6b13-14), son distinguidos de tales sustantivos por Aristóteles en virtud de que aquéllos denominan las propiedades particulares que inhieren en un sujeto portador. La relatividad característica de los parónimos tiene que ver con que tales expresiones sirven para describir al sujeto como portador de una propiedad; ellos no denominan primeramente la propiedad, sino al sujeto calificado por tal propiedad. Así, este texto puede valorarse como una prueba más a favor de la admisión por parte de Aristóteles de accidentes individuales o particulares no sustanciales. ${ }^{39}$ Pero aquí no es éste el tema que me ocupa, sino la circunscripción de la paronimia como una distinción semántica que le permite a Aristóteles explicar los accidentes y dar cuenta del modo en que existen en un sujeto las propiedades no sustanciales a través de la modificación que expresa lingüísticamente esta clase de términos. Entonces, podemos precisar la noción de accidente a partir de la de paronimia:

\footnotetext{
${ }^{39} \mathrm{Si}$ esta prueba se sostiene, habrá que atribuir a Aristóteles — como creo que es correcto hacerlo- la tesis de los accidentes individuales en la categoría de la posición; y de allí también se puede estimar que el estagirita defiende una concepción homogénea en este contexto, o sea, acepta que hay accidentes individuales en todas las categorías. No veo por qué una relación en que se halla una sustancia individual no puede ser individual, y sí, en cambio, su posición, su lugar, su afección, etc. En contra de esto, cfr. Moravcsik 1967, p. 89. Para la relación $x R y$ valdría lo mismo que Moravcsik (p. 87) destaca acerca de la relación de otros accidentes particulares, pues mientras el lenguaje es el mismo para hacer referencia a la relación que guardan múltiples individuos sustanciales, el correlato real debe ser, en esos casos, tan individual como los miembros involucrados en cada relación determinada.
} 
(16) $a$ es un accidente de $x$ si

(i) existe un $A$ cuyo nombre es ' $A$ ';

(ii) ' $a$ ' es parónimo de ' $A$ ';

(iii) ' $a$ ' designa el particular $a^{*}$;

(iv) $a^{*}$ es-en $x$ como en un $S^{*}$.

Dancy ${ }^{40}$ remite a Cat. 11, 14a16-19 para apoyar su idea de que Aristóteles habla de parónimos —o de nombres de parónimos- como designadores de entidades que existen en un sujeto y que no designan primeramente al sujeto ni la propiedad, sino al sujeto en su modo contingente de presentarse. Pero en ese pasaje Aristóteles usa nombres abstractos de contrarios (nósoshygeía, leukótes-melanía, dikaiosýne-adikía) para sostener que los contrarios siempre están en el mismo género, o en géneros contrarios, o ellos mismos son géneros (14a19-20, cfr. la explicación en 14a20-25); o sea que en dicho texto no aparecen parónimos como indicadores de entidades inherentes en un sujeto o en una sustancia. Sin embargo, la afirmación de (16iv) está asegurada por la tesis acerca de la inherencia de los accidentes, explicados en Cat. 2 mediante la predicación ser-en. En cambio, 8, 8b25, 9a31-35, 9b23-24 - también aducidos por Dancy como pruebas documentales de su interpretación- permiten confirmar (16i-iv), pues Aristóteles sostiene que sólo en virtud de que existen cosas calificadas puede aceptarse que existe una cualidad $A$. Sólo hay cualidades en cuanto que existen cosas calificadas, y existen cosas calificadas en la medida en que las cualidades sufren la modificación ontológica que lingüísticamente expresan los parónimos. Las cosas calificadas $\left(x-a^{*}\right)$ tienen una denominación proveniente del adjetivo abstracto $(A)$, tal como lo explica Aristóteles considerando la semántica de los parónimos. Si bien es conducente considerar que el lenguaje platónico de la participación de $x$ en $F$ o las expresiones asociadas - según las cuales $x$ es $F$ en virtud de su participación en la $F$-idad-constituyen antecedentes de esta tesis, la metafísica aristotélica ejecuta una inversión de la relación entre el particular y la propiedad según la cual el particular se denomina de esta u otra manera. La paronimia desempeña un papel central en esa inversión ontológica, pues permite explicar que sólo mediante una adecuada modificación ontológica las propiedades accidentales que especifican la altura, el color, etc., pueden entablar una relación con el sujeto, siendo que, además, sólo existen en virtud de esa relación.

Ahora bien, si esta interpretación es correcta, la tesis de los accidentes particulares es requerida por la teoría aristotélica de los accidentes, ya que la particularización de la propiedad $A-$ i.e. la modificación por la cual $A$ no existe sino en cuanto que $a^{*}$ de $x$ - es lo que corresponde a la interpretación que hace Aristóteles de la modificación lingüística en el nombre de ' $A$ '

${ }^{40}$ Cfr. Dancy 1975, p. 362. 
mediante el parónimo. Dicha particularización en la propiedad que pasa a tener un sujeto individual es requerida por la misma naturaleza individual de $x$. Esta vinculación entre la paronimia y la asunción de particulares no sustanciales no exige que los parónimos ('blanco', 'alto', etc.) expresen la individualidad mediante alguna forma lingüística adecuada (e.g. indexación temporo-espacial). Los parónimos constituyen un recurso del lenguaje natural al que no se le puede exigir razonablemente que refleje con precisión una teoría o una interpretación de la ontología mediante la que se los explica. Incluso en el nivel morfológico de los lenguajes naturales existen variadas dificultades con las expresiones parónimas, como Dancy lo señala acertadamente. ${ }^{41}$ Pero tales dificultades no alteran la tesis de que los accidentes que existen en individuos sustanciales deben ser designados no mediante un adjetivo abstracto, sino sólo mediante los parónimos respectivos. Esto implica cierta particularización de la propiedad (el blanco de Sócrates, la sabiduría de Platón, la altura de Calias, etc.) que no puede equipararse a la especificación del género, ya que aquí se trata de la inherencia de las propiedades que expresa la paronimia.

La distinción aquí sugerida entre (i) particularización de $A$-entendida en términos de un accidente individual $\left(a^{*}\right)$ inherente en sentido fuerte, uno de cuyos rasgos reside en la irrepetibilidad-y (ii) especificación última de $A$ - tomada como una propiedad inherente cuya individualidad, entendida en términos de indivisibilidad, no implica su unidad numérica ni su dependencia estricta de un único sujeto sustancial, numéricamente uno él mismo- va en sentido contrario a la heterodoxa lectura de Owen. Como ya mencioné, este autor sostuvo que los accidentes particulares son tales meramente en cuanto que están específicamente determinados, lo que no excluye que un mismo matiz de verde o una misma magnitud o cierta relación o cierto conocimiento gramatical se presenten en múltiples sujetos numéricamente diferentes. Pero Owen no distingue (i) la inherencia de un accidente universal (e.g. color) respecto de (ii) la inherencia de un accidente particular (este rojo), al sostener que la particularidad de un accidente (sólo) involucra que éste no se predica de un sujeto. ${ }^{42}$ Esta interpretación deja sin ninguna explicación la particularidad de los accidentes no universales. Si Aristóteles sostuviera una tesis como la que Owen le atribuye, su distinción entre color, rojo y el rojo de esta manzana sería excesiva e implausiblemente delgada e insolvente.

Sin embargo, Owen ${ }^{43}$ cree que la identidad específica de los accidentes incluye la unidad numérica; pero con ello establece una relación peculiar entre identidad de accidentes y sustancias, suponiendo dos nociones diferentes de identidad. Es evidente que la identidad específica que existe entre

${ }^{41}$ Cfr. Dancy 1975, pp. 362 s.; Lewis 1991, p. 63.

${ }^{42}$ Cfr. Owen 1965, p. 99.

${ }^{43}$ Cfr. Owen 1965, p. 104. 
Sócrates y Calias no prescribe asumir que ambos son numéricamente uno y el mismo. ¿Cómo hay que entender la singularidad de las propiedades no sustanciales en relación con su inherencia en un sujeto sustancial numéricamente uno? Además, si aceptamos que hay accidentes individuales, ¿nos comprometemos con una errónea objetualización de las propiedades, en cuanto que con aquella aceptación estaríamos admitiendo para la identidad de las propiedades criterios de identificación propios de lo que Aristóteles llama sustancias y actualmente solemos denominar objetos? ¿Cuáles son los criterios de identidad válidos para objetos y cuáles los aplicables a propiedades que nos salvaguardan de incurrir en errores categoriales? ¿Cómo contamos objetos, y cómo lo hacemos con propiedades? Éstas son las cuestiones que, a mi juicio, pueden permitirnos plantear con mayor claridad el problema de la interpretación de los particulares no sustanciales aristotélicos. En lo que resta de este artículo intentaré perfilar una respuesta a los interrogantes anteriores.

\section{Dependencia ontológica, inseparabilidad e individualidad de los accidentes}

Para empezar, no habría que perder de vista que la paronimia da cuenta de la dependencia característica de las entidades no sustanciales; una relación que no puede confundirse —en contra de lo que sugiere Owen- ${ }^{44}$ con el vínculo intrínseco a la misma naturaleza peculiar de los relativos (cfr. Cat. 7, 8a34-b15). En efecto, la inclusión del sujeto determinado -en el cual inhiere un accidente particular - en la determinación completa de un accidente particular no equivale a que la naturaleza de ese accidente no sea otra cosa que la relación que mantiene con el sujeto, como es el caso de los relativos (8a39-b1), y menos que el sujeto sustancial se relacione con sus accidentes como si fuese relativo a ellos, tal como lo requiere la lógica recíproca de los relativos. Así, resulta adecuada la crítica de Allen, ${ }^{45}$ que señala una distinción, descuidada por Owen, entre depender de y ser relativo a. Esta distinción restituye a los accidentes, incluidos los particulares, su propia determinación específica proveniente del hecho de que son instancias de una especie de color (o especies de un color), de una especie de cantidad (o especies de una cantidad) e incluso instancias de una clase de relación (o especies de una relación), por lo cual los accidentes poseen una determinación específica expresable en su definición. Tal es la clase de determinación independiente propia de cualquier accidente, considerando un accidente bajo su determinación específica, lo que hace que un accidente sea algo por sí mismo y, por tanto, distinto de un

${ }^{44}$ Cfr. Owen 1965, p. 101.

${ }^{45}$ Cfr. Allen 1969, pp. 33 s. 
relativo, ya que la identidad de un relativo consiste en su relación con otra cosa. Así, la advertencia de Owen no nos impide seguir sosteniendo la particularidad de los accidentes, tal como ésta se halla sugerida en la interpretación que he propuesto de la paronimia, ni nos lleva a despojar a los accidentes de su carácter ontológico intrínsecamente dependiente, en cuanto que son-en una sustancia. En síntesis, la particularización de una propiedad accidental mantiene la identidad específica de dicha propiedad sin que ello implique dos extremos opuestos: por un lado, abolir la dependencia propia de las entidades que son-en, y, por el otro, convertir la dependencia característica de un accidente individual en la peculiar de los relativos.

Aristóteles usa, entonces, su noción de paronimia para aclarar también el modo de existencia de los accidentes. Esto puede confirmarlo un texto como el de Tópicos II 2, 109b4-12. Allí, Aristóteles pretende aclarar que en un enunciado como "(un) blanco es(tá) coloreado" no se atribuye el género color al color particular blanco, pues quien formula ese enunciado articula morfológicamente el predicado mediante un parónimo. Si, en cambio, el predicado estuviese representado por el término 'color' (chró$m a$ ), entonces se trataría de un enunciado en el que se predicaría sinónimamente del sujeto el contenido conceptual del predicado (cfr. 109b56). Aristóteles explica a continuación, teniendo en cuenta la doctrina de los predicables, que coloreado tampoco puede clasificarse como una definición o un propio de blanco, ya que cualquiera de estos dos últimos tipos de predicado se dicen de una cosa determinada y de ninguna otra específicamente diferente, mientras que coloreado se atribuye igualmente a cosas diferentes, como a una madera, a una piedra, a un hombre o a un caballo (109b11-12). Salta a la vista que Aristóteles interpreta el parónimo como una morfología que se usa para designar un accidente de un sujeto (cfr. 4, 111a33-b11); el parónimo no es un nombre (un sustantivo) que se atribuye al sujeto, sino una expresión que sirve para designar la modificación en la presentación de un objeto cuyo núcleo de determinación -aquello que se delimita mediante la predicación sinónima de términos como 'hombre' en "Sócrates es un hombre"- permanece inalterable tras esa modificación.

Esto permite entender que la dependencia respecto del sujeto que entrañan las entidades designadas mediante parónimos es muy fuerte o, estrictamente, de otro tipo en comparación con la que Aristóteles admite para las sustancias segundas, ${ }^{46}$ ya que los parónimos designan, en reali-

\footnotetext{
${ }^{46}$ Cat. 5, 2a36-b3, expresa la dependencia que en relación con las sustancias primeras cabe tanto a las sustancias segundas como a las entidades no sustanciales. La intención del pasaje es subrayar la dependencia de ambas clases de entidades respecto de la sustancia primera, lo que constituye un paralelo entre tales entidades - no obstante, la dependencia de ambas es diferente, según corresponde a la distinta clase a la cual pertenece cada una de ellas-. Acep-
} 
dad, no una cosa aparte del portador, sino el portador modificado accidentalmente. Esto implica también que lo que es-en un sujeto, en cuanto que se halla presente actualmente en él, o sea, en cuanto que constituye una modificación (contingente) en la presentación del sujeto, no puede existir separado de ese sujeto, ya que tal modificación es parte de la manera en que se presenta el sujeto particular. ${ }^{47}$ Para las cosas que se dicen-de un sujeto puede haber, en principio, más de un sujeto, de tal manera que no dependen de un único y determinado ejemplar de la clase para existir; en cambio, las cosas que son-en dependen de un único sujeto particular y no pueden existir aparte de él, ni existir en cualquier sujeto numéricamente diferente de aquel en el cual existen, ya que los accidentes no guardan con su sujeto una relación universal. Esto no entraña ese error categorial por el cual asociamos la identidad de una propiedad a su irrepetibilidad, error en el que se incurre aplicando a propiedades un criterio de identidad peculiar de objetos. La tesis de Aristóteles es que la inseparabilidad fuerte de un accidente particular — proveniente de la misma inherencia - implica su irrepetibilidad; y esto no contradice que el accidente que se presenta en sujetos numéricamente diferentes sea el mismo, aunque no puede ser uno y el mismo. ${ }^{48}$

Retomando el ejemplo de los Tópicos, 'coloreado' designa el mismo sujeto que 'blanco' como entidad-soporte, pero con una modificación accidental diferente: es una cosa — que puede ser de distinto tipo, pues la predicación accidental no restringe la correlación entre sustrato y propiedad a la pertenencia de ambos a una misma especie o a un mismo género- blanca

to la reconstrucción del argumento sobre la dependencia de universales sustanciales respecto de sustancias primeras hecha por Moravcsik (1967, pp. 94 s.): en 2a36-37, el antecedente ("(i) tal como el animal se predica del hombre") no da la razón que fundamenta la conclusión de 2a37-38 ("(iii) así también (animal se predica) de cierto hombre (individual)”). Esa razón es provista por que "(ii) el hombre se predica de cierto hombre (individual)". La transitividad se halla presupuesta en (ii) y por ello se sigue la conclusión del argumento en (iii). Sin embargo, la tesis de este pasaje es la prioridad del sujeto que explica la dependencia de —en este caso- las sustancias segundas; tal prioridad no se obtiene, empero, de la sola transitividad aportada por (ii), sino del concepto completo de las sustancias individuales o primeras.

${ }^{47}$ Por tanto, en contra de las opiniones de Owen 1965, p. 104, y Frede 1987a, p. 59, un accidente particular no es meramente inseparable de algún sujeto, sino de ese sujeto en el que se presenta. Cfr. Allen 1969, pp. 35 s. Esta tesis es la interpretación correcta de la condición de inseparabilidad fuerte, formulada en Cat. 2, 1a25.

${ }^{48}$ Para una discusión de la interpretación de Frede sobre Cat. 1a24-25, cfr. Devereux 1992, pp. 119 ss., y 126. Para Devereux, la dependencia de un accidente con respecto al individuo sustancial en el cual inhiere no implica que ese accidente no pueda existir en otro sujeto individual como el mismo accidente. Devereux explica el hecho de que un accidente particular no puede ser en un individuo sustancial diferente de aquel en el cual actualmente existe como si ello fuera una mera consecuencia de la inseparabilidad que caracteriza a los accidentes, algo que no tendría incidencia en la particularidad de los mismos. Mi propuesta es, en cambio, que la inseparabilidad de los accidentes incide de manera condicionante en su irrepetibilidad, y que esta última explica la particularidad de aquéllos. En contra de una lectura como la de Devereux, cfr. Allen 1969, p. 31. 
la que aparece coloreada puesto que tiene (un) color. El parónimo designa, así, un compuesto accidental cuyos elementos son el sujeto más su accidente, y un compuesto accidental se entiende como la presentación de un objeto en su modificación contingente. Cuando, para recoger el ejemplo de Dancy, ${ }^{49}$ un químico en el laboratorio le indica a su colega que inspeccione una materia bajo el microscopio y le informa que la cosa en que está interesado es coloreada, ni el colega precisa conocer la morfología y hacer alguna conjetura acerca de la estructura molecular de la cosa que busca quien da esa indicación, ni quien da esa indicación supone que quien observa a través del microscopio conoce dicha morfología o tiene una idea cierta y pormenorizada acerca de la presentación general del material observado (lo que conllevaría conocer otras propiedades del mismo, eventualmente tan vagas para la clasificación como puede ser el color por sí mismo). Para que se genere una respuesta adecuada a las expectativas del jefe de laboratorio de parte de su asistente es suficiente con que éste - sin sobrecargar con ello ontológicamente su praxis con una teoría de los accidentes- reaccione adecuadamente ante la percepción de algo coloreado bajo el microscopio señalando, es decir, individualizando una cosa coloreada que puede ser la que buscaba su colega. El ejemplo es bastante dúctil como para mostrar que una indicación dada mediante un parónimo no presupone el conocimiento o la determinación de la estructura de algo -qué es lo coloreado que busca el jefe de laboratorio puede resultar, tras el descubrimiento de algo coloreado bajo el microscopio, todavía completamente incierto para uno y otro colega (cfr. Cat. 5, 2b32-37) - ni el hallazgo de una cosa coloreada ofrece necesariamente una respuesta directa o suficiente acerca de esa estructura. Más bien, enunciados que incluyen parónimos en el lugar del predicado tienen un contenido semántico que parece apropiadamente aclarado por la idea aristotélica de los compuestos accidentales.

Tal vez la teoría completa de los compuestos accidentales requiere una base empírica más amplia y, en tal sentido, aquellos enunciados, tomados por sí mismos, resultan sobredeterminados por esta teoría. Sin embargo, Aristóteles no dispone únicamente de esos enunciados como base empírica de su teoría. ${ }^{50}$ En efecto, esos enunciados designan la presentación

\footnotetext{
${ }^{49}$ Cfr. Dancy 1975, p. 364.
}

${ }^{50}$ Dancy sostiene que un sujeto o una sustancia puede ser considerada como parónima en cuanto se le aplica el nombre de una propiedad que es un parónimo y se toma el sujeto qua portador de esa propiedad. "Calias sólo puede ser un parónimo de justicia en cuanto (un) justo" (Callias can only be a paronym from justice as (a) just (thing); cfr. Dancy 1975, p. 365 (los paréntesis son del autor)). Sin duda, esta descripción de Calias qua justo y, en tal sentido, como un parónimo, no entraña un problema acerca de la clase de entidad que Calias es (una sustancia), ya que Calias-justo (i.e. Calias descrito como justo) no es una descripción alternativa de Calias como tal (per se) —ni, por ende, puede sustituir al nombre de Calias en cualquier enunciado salva intensione-, sino que corresponde a una descripción accidental 
de algo de una determinada manera, sin vincular necesariamente y siempre tal presentación con una determinada estructura de la cosa. Mediante la expresión "busco algo coloreado" puedo proponerme indicar al oyente, más bien, cómo aparece algo de cierta manera que puede hacerlo individualizable - aunque un accidente no permite individualizar necesaria e infaliblemente un objeto- ${ }^{51}$

de Calias (per accidens) y, en esa medida, dicha descripción no designa el sujeto, sino el compuesto accidental del que forman parte el sujeto y uno de sus accidentes. C fr. la discusión de Dancy 1975, pp. 365 ss. (cfr. Top. V 4, 133b15-24, 31-37), del silogismo quineano:

(i) (Algo/un) justo es un quale,

(ii) Calias es (algo/un) justo,

(iii) Calias es un quale.

¿Es, en realidad, referencialmente opaca en el lugar del sujeto la primera premisa? ¿Es ésa la razón por la cual se llega a la conclusión inválida de la conclusión? No tengo espacio aquí para discutir esta cuestión (para ello cfr. Lewis 1991, capítulos 3-5); me limitaré a señalar mi interpretación para redondear el tratamiento que ahora hago de los parónimos. De Top. V 4 puede concluirse - aunque la interpretación no está exenta de controversias- que Aristóteles no considera a 'Calias justo' y a 'Calias' como dos entidades diferentes sin más (cfr. 133b3133), pero tampoco admite que 'Calias justo' (a lo cual Aristóteles hace referencia mediante la expresión "el accidente tomado junto con aquello en lo que acontece (el accidente)") es meramente una descripción alternativa de Calias, con lo cual ambas expresiones servirían por igual para designar la misma cosa. Un parónimo ('el justo') o la expresión explícita que incluye el nombre del sujeto ('Calias justo') designa una entidad que es diferente del sujeto en cuanto al ser, ya que no es lo mismo para el hombre ser hombre y para el hombre blanco ser hombre blanco —esto se aplica obviamente también a justo- (tò eînai, 133b34, 35; Física (Ph.) I 7, 190a1-17, b23-191a3). Así, no se trata de opacidad referencial en el parónimo '(algo/un) justo', sino de un contenido semántico diverso que pertenece a ese parónimo respecto del que se tiene en '(un) hombre' o 'Calias', y eso es lo que explica la invalidez que para Aristóteles tiene la conclusión "(un) hombre/Calias es un quale". La distinción en cuanto al ser es lo que debe bloquear la conclusión sofística que pretende forzarnos a aceptar (iii) sobre la base de que (a) Calias y (b) (algo/un) justo no son cosas diferentes. Para Aristóteles (cfr. APo. I 22, 83a25-32), la diferencia en el ser entre el sujeto y el compuesto accidental se explica por el hecho de que un accidente, en el propio contenido semántico de su nombre o en su propia determinación, mantiene una dependencia respecto de un sujeto que es, precisamente, diferente; y en virtud de esa misma diferencia no puede identificarse el compuesto accidental con el sujeto. Cuadra a la economía de la metafísica aristotélica bloquear esta multiplicación de entidades mediante una distinción en cuanto a la independencia ontológica, por la cual no todo predicado con sentido corresponde a una entidad separada que debe contarse como una cosa más en el mundo. En este contexto, cosas cuyo nombre no se deriva de un nombre abstracto, como los parónimos, se cuentan entre las que Aristóteles acepta como básicas.

${ }^{51}$ En Top. I 9, 103b27-35, Aristóteles considera qué sucede si se aplica la pregunta ¿qué es? a predicados que no dan el qué es (la esencia) de algo (e.g. ¿qué es blanco?). Lo que se obtiene es una indicación acerca del género y, aplicando nuevamente la misma clase de pregunta al género, se obtiene el tipo de entidad bajo el cual tiene que clasificarse esa clase de cosas (e.g. cualidad). 


\section{BIBLIOGRAFÍA}

Se citan las obras de Aristóteles según las ediciones de Oxford Classical Texts.

\section{Comentarios}

Ackrill, John L., 1963, Aristotle's Categories and De Interpretatione, trad. y notas de J.L. Ackrill, Oxford University Press, Oxford (Clarendon Aristotle Series).

Barnes, Jonathan, 1993, Aristotle Posterior Analytics, 2a. ed., trad. y comentario de J. Barnes, Oxford University Press, Oxford (Clarendon Aristotle Series).

Detel, Wolfgang, 1993, Aristoteles, Analytica Posteriora, traducido y comentado por W. Detel, 2 vols., Aristoteles Werke en trad. alemana, fundada por E. Grumach, ed. H. Flashar, 3/II, Akademie Verlag, Berlín.

Oehler, Klaus, 1997, Aristoteles, Kategorien, traducido y comentado por K. Oehler, Aristoteles Werke en trad. alemana, fundada por E. Grumach, ed. H. Flashar, 1/I, Akademie Verlag, Berlín.

Ross, W.D., 1949, Aristotle's Prior and Posterior Analytics, texto revisado, introd. y comentario de W.D. Ross, Oxford University Press, Oxford.

Simplicio, 1907, In Aristotelis Categorias commentarium, ed. Kalbfleisch, Commentaria in Aristotelem Graeca VIII, Reimer, Berlín.

Índice

Bonitz, Hermann, 1955 (1870), Index Aristotelicus, 2a. ed., Akademische und Verlagsanstalt, Graz.

\section{Literatura específica}

Ackrill, John, 1972-1973, "Aristotle's Definitions of Psyche", Proceedings of the Aristotelian Society, vol. 73, pp. 119-133.

Allen, R.E., 1969, "Individual Properties in Aristotle's Categories", Phronesis, vol. 14, pp. 31-39.

Chen, Chung-Hwan, 1957, “On Aristotle's Two Expressions: kath'hypokeiménou légesthai and en hypokeiménoi eînai. Their Meaning in Cat. 2, 1a20-b9 and the Extension of This Meaning", Phronesis, vol. 2, pp. 148-159.

Code, Alan, 1986, "Aristotle on Essence and Accident", en R.E. Grandy y R. Warner (comps.), Philosophical Grounds of Rationality, Oxford University Press, Oxford/Nueva York, pp. 411-444.

_ 1985, "On the Origins of Some Aristotelian Theses about Predication", en J. Bogen y J.E. McGuire (comps.), How Things Are, Reidel, Dordrecht/Boston, pp. 101-131.

Dancy, Russell M., 1975, “On Some of Aristotle's First Thoughts about Substances", The Philosophical Review, vol. 84, pp. 338-373.

Detel, Wolfgang, 1998, "Eine terminologische Rekonstruktion von Arist. Cat. 1-5", en R. Enskat (comp.), Amicus Plato magis amica veritas, Homenaje a Wolfgang Wieland, W. de Gruyter, Berlín/Nueva York, pp. 60-81.

Devereux, Daniel T., 1992, "Inherence and Primary Substance in Aristotle's Categories", Ancient Philosophy, vol. 12, pp. 113-131. 
Driscoll, John A., 1981, "EIDE in Aristotle's Earlier and Later Theories of Substance", en D.J. O'Meara (comp.), Studies in Aristotle, The Catholic University of America Press, Washington, D.C., pp. 129-159.

Duerlinger, James, 1970, "Predication and Inherence in Aristotle's Categories", Phronesis, vol. 15, pp. 179-202.

Ebert, Theodor, 1998, "Aristotelian Accidents", Oxford Studies in Ancient Philosophy, vol. 16, pp. 133-159.

Frede, Michael, 1987a, "Individuals in Aristotle", en Frede 1987c, pp. 49-71.

— $1987 \mathrm{~b}$, "The Title, Unity, and Authenticity of the Aristotelian Categories", en Frede 1987c, pp. 11-28.

—_, 1987c, Essays in Ancient Philosophy, University of Minnesota Press, Minneapolis.

Gill, Mary Louise, 1991, Aristotle on Substance: The Paradox of Unity, Princeton University Press, Princeton.

Graham, Daniel W., 1999, Aristotle's Two Systems, Oxford University Press, Nueva York.

Hartmann, Edwin, 1977, Substance, Body, and Soul, Aristotelian Investigations, Princeton University Press, Princeton.

Heinaman, Robert, 1981, "Non-Substantial Individuals in the Categories", Phronesis, vol. 26, pp. 295-307.

Irwin, Terence H., 1992, Aristotle's First Principles, Oxford University Press, Nueva York.

Jones, Barrington, 1975, "An Introduction to the First Five Chapters of Aristotle's Categories", Phronesis, vol. 20, pp. 146-172.

— 1972 , "Individuals in Aristotle's Categories", Phronesis, vol. 17, pp. 107-123.

Lewis, Frank, 1991, Substance and Predication in Aristotle, Cambridge University Press, Nueva York.

Loux, Michael J., 1991, Primary Ousia. An Essay on Aristotle's Metaphysics $Z$ and H, Cornell University Press, Ithaca.

Lowe, E.J., 1989, Kinds of Being. A Study of Individuation, Identity and the Logic of Sortal Terms, Oxford University Press, Nueva York (Aristotelian Society Series, 10).

Marcus, Ruth Barcan, 1971, "Essential Attribution", The Journal of Philosophy, vol. 48, pp. 187-202.

Mié, Fabián, en prensa, "Sustancia y predicación en las Categorías de Aristóteles", Elenchos.

Moravcsik, J.M.E., 1967, "Aristotle on Predication", The Philosophical Review, vol. 76, pp. 80-96.

Owen, G.E.L., 1965, "Inherence", Phronesis, vol. 10, pp. 97-105.

— 1960 , "Logic and Metaphysics in Some Earlier Works of Aristotle", en I. Düring y G.E.L. Owen (comps.), Aristotle and Plato in the Mid-Fourth Century, trabajos del Symposium Aristotelicum realizado en Oxford, agosto de 1957, Studia Graeca et Latina Gothoburgensia XI, Gotenburgo, pp. 163-190.

Rapp, Christof, 1995, Identität, Persistenz und Substantialität, Untersuchungen zum Verhältnis von sortalen Termen und Aristotelischer Substanz, Symposion 103, Karl Alber, Munich/Friburgo de Brisgovia. 
Sellars, Wilfrid, 1985, "Towards a Theory of Predication”, en J. Bogen y J.E. McGuire (comps.), How Things Are, Reidel, Dordrecht/Boston, pp. 285-322.

Strawson, P.F., 1963, Individuals, An Essay in Descriptive Metaphysics, Anchor Books, Garden City.

—_ 1953/1954, "Particular y general”, Ensayos lógico-lingüísticos, trad. A. García Suárez y L.M. Valdés Villanueva, Tecnos, Madrid, 1983, pp. 40-66.

Wedin, Michael V., 2000, Aristotle's Theory of Substance. The Categories and Metaphysics Zeta, Oxford University Press, Nueva York.

Recibido el 6 de abril de 2005; aceptado el 25 de agosto de 2005. 